\title{
ON THE UNIQUENESS CONDITIONS AND BIFURCATION CRITERIA IN COUPLED THERMO-ELASTO-PLASTICITY
}

\author{
Z. ŚLODERBACH \\ Opole University of Technology \\ Faculty of Applications of Chemistry and Mechanics \\ 45-036 Opole, Luboszycka 7, POLAND \\ E-mail: z.sloderbach@po.opole.pl
}

\begin{abstract}
The global and local conditions of uniqueness and the criteria excluding a possibility of bifurcation of the equilibrium state for small strains are derived. The conditions and criteria are derived analyzing the problem of uniqueness of solution of the basic incremental boundary problem of coupled generalized thermo-elastoplasticity. This paper is a continuation of some previous works by the author, but contains new derivation of the global and local criteria excluding a possibility of bifurcation of the equilibrium state for a comparison body dependent on statically admissible fields of stress velocity. All the thermal elastoplastic coupling effects, nonassociated laws of plastic flow and influence of plastic strains on thermoplastic properties of a body were taken into account in this work. Thus, the mathematical problem considered here is not a self-conjugated problem. The paper contains four Appendices A, B, C and D where the local necessery and sufficient conditions of uniqueness have been derived.
\end{abstract}

Key words: bifurcation of the equilibrium state, conditions and criteria of uniqueness, boundary-value problem, generalized coupled thermo-elasto-plasticity, comparison bodies, family of local conditions of uniqueness, parameter of the optimization.

\section{Introduction}

The incremental boundary-value problem of generalized coupled thermoplasticity will be formulated. This will be followed by an interpretation of the uniqueness conditions for the solution of that problem. The necessary uniqueness conditions will be derived as well as the sufficient local condition, the sufficient global uniqueness condition and the sufficient global criterion precluding state of the bifurcation. A similar incremental boundary problem of coupled generalized thermoplasticity has been previously investigated and discussed in (Śloderbach, [1], [2], [3]). In this paper, necessary and sufficient conditions of uniqueness of solution of formulated incremental boundary-value problem of coupled generalized thermoplasticty for the case of small gradients of displacements (small strains) are derived. The global conditions and local conditions of uniqueness are derived. The uniqueness conditions for the generalized coupled thermoplastic body and suitable comparison bodies (Mróz and Raniecki, [4], [5]; Raniecki, [6]; Śloderbach, [1], [3], [7]) were derived. The derived global and local uniqueness conditions are suitable necessary and sufficient conditions excluding the occurrence of the bifurcation state of equilibrium in coupled generalized thermoplasticity and also in isothermal loading processes. We prove that the local uniqueness conditions for the generalized thermo-elastoplastic body and the comparison bodies are same, but the methods of calculating the bifurcation state (using the global sufficient uniqueness condition) for the case of comparison bodies are less complicated (there exists a linear dependence between the stress rate and strain rate), see (Mróz and Raniecki, [4], [5]; Raniecki, [6]; Śloderbach, [1], [3], [7]). Thus, introduction of such comparison bodies seems to be advisable. The global uniqueness conditions for generalized coupled elasticplastic solids and the global criteria excluding bifurcation for comparison bodies are different. This work is a continuation of the work (Śloderbach, [8]), devoted to the coupling effects in generalized thermo-elastoplasticity for the case of small deformations. 
In a generalized case, the constitutive equations of coupled thermoplasticity are non-associated laws of plastic flow, even if Gyarmati's (Gyarmati, [9]) postulate is assumed (see Śloderbach, [1], [3], [7], [8]). They also include the effects of thermomechanical coupling effects and take into account the elastic-plastic conjugation. It means that they can be applied for the description of not only metallic bodies, but porous materials, sintered powders, rocks and soils, as well. The paper also presents some special cases of the global and local conditions of uniqueness for more specific models of bodies. In such less general models, constitutive functions occurring under conditions of uniqueness take simpler forms.

The derived conditions of uniqueness seem to be important from both mathematical and practical point of view. They are a device for the estimation of critical loads. If the critical loads are exceeded, bifurcation of the equilibrium state is possible. In comparison to the previous papers about the uniqueness of solution and bifurcation of equilibrium states (Śloderbach, [1], [3], [7]; Mróz and Raniecki, [4], [5]; Raniecki, [6];), this paper presents a derived new comparison body dependent on statically admissible fields of stress rate. For such a body a sufficient local and global uniqueness conditions were derived, developing problems discussed in the above mentioned papers. Derived sufficient local and global uniqueness conditions are simultaneously local and global criteria excluding the appearance of the bifurcation state. The uniqueness conditions and bifurcation criteria derived in Śloderbach [1], [3], Mróz and Raniecki [4], [5] Raniecki, [6] were related to the comparison body depending on kinematically admissible fields of strain rate.

In order to analyze uniqueness of the boundary problem solution, it is assumed that a thermodynamic state of the body in a certain moment of the body plastic deformation is known. However, it is necessary to determine velocity fields of strain and displacement as well as temperatures for the input values of stress and divergence of a vector of the flux of heat exchanged by the surface through the elementary area [see the problems $b_{1}$ and $b_{2}$ considered in this paper and in Sloderbach [1], [3], [7].

Papers on non-isothermal thermo-elasto plasticity and isothermal elasto-plasticity with associated and non-associated laws of plastic flow in the range of large displacements have been published in recent twenty years, (see e.g. Armero and Simo J.C. [10]; Benall and Bigoni, [11]; Bertram, [12]; Candija and Brnic, [13]; Casey, [14]; Epstein and Maugin, [15]; Hakansson et al., [16]; Huttel and Matzenmiller, [17]; Itskov, [18]; Lehmann, [19]; Mahnken and Stein, [20]; Marakin and Sokolova, [21]; Maugin and Epstein,, [22]; Meyers et al., [23]; Miehe, [24]; Srinivasa, [25]; Xiao et al. [26]; Nguyen, [27]; Śloderbach and Pajak, [28], [29]; Śloderbach, [7], [8], [30]). The constitutive equations and plasticity conditions for isotropic and anisotropic materials were analyzed in these papers; the methods of numerical calculations were also used. In some works, (e.g. Bertram, [12]; Candija and Brnic, [13]; Casey, [14]; Epstein and Maugin, [15]; Hakansson et al., [16]; Huttel and Matzenmiller, [17]; Itskov, [18]; Mahnken and Stein, [20]; Marakin and Sokolova, [21]; Maugin, [31]; Maugin and Epstein, [22]; Meyers et al. [23]; Simo and Miehe, [32]; Miehe, [24]; Srinivasa, [25]; Xiao et al. [26]) the problems of description of large thermo and elasto-plastic deformations and taking appropriate measures for deformations, additivity of elastic and plastic deformations and problems related to actual and relative configurations were considered. In papers (Mahnken and Stein, [20]; Meyers et al., [23]) the topics of linear and non-linear kinematic and isotropic reinforcement were analyzed. Also the problems of stability and behaviour under thermo-mechanical and mechanical stresses, (see e.g. Benall and Bigoni, [11]; Candija and Brnic, [13]; Epstein and Maugin, [15]; Lehmann, [19]; Marakin and Sokolova, [21]; Simo and Miehe, [32]; Srinivasa, [25]) were analyzed. The majority of the papers describing large deformations in thermo-elastoplasticity use the method of inner parameters in the frame of thermodynamics of non-reversible processes.

In this part of the paper the incremental boundary problem of coupled geneneralized thermo-elastoplasticity has been being formulated. Next, conditions of uniqueness of solution of the formulated incremental boundary problem have been interpreted. The required conditions of uniqueness of the problem solution have been derived. Next, a sufficient local condition and global sufficient conditions of uniqueness of the problem solution have been formulated for a body model of the generalized thermo-elasto-plasticity, and for comparative bodies dependent on kinetically acceptable fields of plastic strains and statically acceptable stress velocity fields (Śloderbach, [1], [3], [7]; Raniecki, [33]; Raniecki and Bruhns, [34]). From the physical point of view the conditions of uniqueness are understood as proper criteria eliminating the occurrence of bifurcation of the equilibrium state. Similar incremental boundary problems of the coupled 
thermo-elasto-plasticity for small and large deformations were tested and discussed in papers, (see e.g. Nguyen, [27]; Raniecki, [6], [33]; Śloderbach, [1], [3], [7]; Śloderbach and Pajak, [28], [29]). This paper uses the data from them. An original element consists in the assumption of non-associated laws of plastic flow and including the influence of plastic strains on thermoelastic properties of bodies and derivation of a reference body dependent on statically acceptable stress velocity fields. Such a requirement leads to a more difficult problem than those previously considered.

The paper (see section 5) contains four Appendices A, B, C and D where the local necessery and sufficient condition of uniqueness of solution of the formulated incremental boundary-value problem of coupled generalized thermoplasticty for the case of small gradients of displacements (small strains) has been derived. This condition is a local criterion excluding bifurcation of the equilibrium state for the derived comparison body dependent on statically admissible fields of stress velocity. It has been shown that the local sufficient condition of uniqueness for the comparison body, resulting from the requirement of positive integrand denoted as $I^{\prime}$ and $J^{\prime}$, is the same as that for the integrand $I$ and $J$ for the generalized coupled thermoplastic body (Śloderbach, [1], [3], [7], [30]). It is a certain criterion proving that the introduction of the reference body and the integrands $I^{\prime}$ and $J^{\prime}-$ being the lower majorant of the expression for the generalized thermoplastic body - seems to be advisable.

In Appendix A, necessary conditions of uniqueness of the solution have been derived for the formulated problems expressed as $\boldsymbol{b}_{1}$ and $\boldsymbol{b}_{2}$ (see sec. 2). This appendix contains also conditions of invertibility of constitutive equations expressed in velocities of stresses and strains.

Appendix B presents a procedure of the proof of the theorem concerning the local sufficient condition of uniqueness formulated in subsection 4.1. The presented proof is a mathematical indirect proof. Investigating a positive definition of the integrand expressed as $I$ (see subpoint 4.2.3), the author derived the mentioned sufficient local condition of uniqueness.

Appendix B presents also a procedure of obtaining the optimum (minimal) condition of uniqueness from the derived one-parameter family of local conditions of uniqueness for the derived comparison body dependent on statically admissible fields of stress velocity. Conditions of uniqueness and estimations of bifurcation states in the theory of plasticity for the case of large strains with associated and non-associated laws of plastic flow, depending on kinematically admissible fields of strain velocity were analyzed in Śloderbach [1], [3], [7], [30], Raniecki [6], Raniecki and Bruhns [34].

In Appendices $\mathrm{C}$ and $\mathrm{D}$, it has been shown that sufficient local conditions of uniqueness result from the requirement of positive definitions of integrands for reference bodies, defined as $J^{\prime}$ and $I^{\prime}$ (see subpoints 4.2.2 and 4.2.3). Those conditions are the same as those for the generalized thermo-elastic-plastic body defined by the functions $J$ and $I$. This mathematical fact is also a certain criterion proving that an introduction of such integrand expressions $J^{\prime}$ and $I^{\prime}$, being some certain majorants of integrand expressions $J$ and $I$ for thermo-elastic-plastic bodies, makes sense.

\section{Uniqueness solution of incremental problems for homogenous processes}

A homogeneous physical body of unit mass is being considered. When the thermodynamic state of each particle of the body is the same at any moment of the process, the process is called homogeneous. In the case of such processes, the quantity divq occurring in the equations for temperature should be understood as the rate of global heat exchange between the physical body and the environment, and $\rho_{0}$ as a reverse of the total volume of the body.

Let us assume that the local thermodynamic state is described by the following parameters of state (Raniecki,[6]; Śloderbach, [1], [2], [7], [8] [30]):

$\varepsilon^{e}$ - tensor of elastic strain, $s$ - specific entropy (per unit mass),

$\boldsymbol{\kappa}^{(M)}$ - the set of symmetric internal tensor parameters of second order $(M=1, \ldots, n)$, $\boldsymbol{\kappa}=\boldsymbol{\kappa}^{T}$, that is $\kappa_{i j}=\kappa_{j i}, \boldsymbol{\kappa}^{(N)}$ - the set of internal scalar parameters $(N=1, \ldots, m)$.

Now the symbol $K$ will denote the set of internal parameters in the form of a vector of pair $K \Leftrightarrow$ $\left\{\boldsymbol{\kappa}^{(M)}, \boldsymbol{\kappa}^{(N)}\right\},(M=1, \ldots n)$ and $(N=1, \ldots, m)$. 
The systems of constitutive thermo-elasto-plastic equations composed of the equation for the temperature and the relation between the rates of elastic strain, elastic stress and temperature, have the following form (Śloderbach, [8])

$$
\begin{aligned}
& \left\{\begin{array}{l}
\rho_{0} c_{\sigma} \dot{T}=\gamma_{0} D-\gamma_{7} T \boldsymbol{\alpha}: \dot{\sigma}+\bar{\gamma}_{8} T \frac{\partial \Pi}{\partial T} \cdot \dot{K}+q_{0}, \\
\dot{\boldsymbol{\varepsilon}}^{e}=\boldsymbol{L} \dot{\boldsymbol{\sigma}}+\gamma_{4} \boldsymbol{\alpha} \dot{T}+\bar{\gamma}_{9} \boldsymbol{Z} \dot{K}
\end{array}\right. \\
& \left\{\begin{array}{l}
\rho_{0} c_{\mathcal{\varepsilon}} \dot{T}=\gamma_{0} D-\bar{\gamma}_{6} T \boldsymbol{\alpha}: \boldsymbol{M} \dot{\boldsymbol{\varepsilon}}^{e}+\bar{\gamma}_{8} T \frac{\partial \Pi}{\partial T} \cdot \dot{K}+q_{0}, \\
\dot{\boldsymbol{\sigma}}=\boldsymbol{M} \dot{\boldsymbol{\varepsilon}}^{e}-\gamma_{5} \dot{T} \boldsymbol{M} \boldsymbol{\alpha}+\bar{\gamma}_{10} \boldsymbol{N} \dot{K},
\end{array}\right.
\end{aligned}
$$

where particular types of thermodynamic, thermomechanical and mechanical (elastic-plastic) couplings are denoted by a Greek letter $\gamma$ with a suitable subscript and superscript $\gamma_{I}$ and $\bar{\gamma}_{i}$, (see Śloderbach, [1], [2], [8]). The symbol $\gamma_{0}$ stands for dissipation heat which is not a thermostatic thermal effect.

Let us assume that the thermodynamic state of the body at a certain moment $t_{0}$ of a homogenous process is known and such that the condition $F_{l}=F=0$ is satisfied. The following incremental problems can be formulated for such type of processes. Satysfying the set of field and constitutive equations of, we must find, for the time $t_{0}$, for the problems $\left(a_{1}, a_{2}, b_{1}\right.$ and $\left.b_{2}\right)$ the values

$$
\begin{aligned}
& a_{1 .}\left(\dot{\varepsilon} \text { and } q_{0}\right) \text { assuming that } \dot{\sigma}\left(t_{0}\right) \text { and } \dot{T}\left(t_{0}\right) \text { are prescribed, } \\
& a_{2 .}\left(\dot{\sigma} \text { and } q_{0}\right) \text { assuming that } \dot{\varepsilon}\left(t_{0}\right) \text { and } \dot{T}\left(t_{0}\right) \text { are prescribed, } \\
& b_{1 .}(\dot{\varepsilon} \text { and } \dot{T}) \text { assuming that } \dot{\sigma}\left(t_{0}\right) \text { and } q_{0}\left(t_{0}\right) \text { are prescribed, } \\
& b_{2 .}(\dot{\sigma} \text { and } \dot{T}) \text { assuming that } \dot{\varepsilon}\left(t_{0}\right) \text { and } q_{0}\left(t_{0}\right) \text { are prescribed }
\end{aligned}
$$

where $q_{0}=-\operatorname{div} \boldsymbol{q}$.

It is easy to see that if a solution of the problems $\left(a_{1}\right)$ and $\left(a_{2}\right)$ is to be unique, it is necessary that the following respective conditions known from the isothermal theory of plasticity should be satisfied (Raniecki, [6], [33]; Śloderbach, [1], [3], [7], [30]; Śloderbach and Pajak [28])

$$
h>0 \quad \text { and } \quad h+\boldsymbol{g}_{p}: \boldsymbol{M F}_{\boldsymbol{\sigma}}>0
$$

where

$$
\boldsymbol{g}_{p}=\boldsymbol{F}_{l, \boldsymbol{\sigma}}+\bar{\gamma}_{9} \boldsymbol{Z} * b
$$

$h$ - is the isothermal strain-hardening function, $\boldsymbol{F}_{\sigma}=\frac{\partial F}{\partial \boldsymbol{\sigma}}$ and $\boldsymbol{F}_{l, \boldsymbol{\sigma}}=\frac{\partial F_{1}}{\partial \boldsymbol{\sigma}}$,

$b$ - is the function describing evolution of internal parameters $\dot{K}$ (Śloderbach, [1], [2], [8], [30]).

For the problems $\left(a_{1}\right)$ and $\left(a_{2}\right)$, conditions (2.3) are also sufficient. However, two solutions of the problems $\left(b_{1}\right)$ and $\left(b_{2}\right)$ may exist, even if the inequalities (2.3) are satisfied. The necessary uniqueness conditions for the problems $\left(b_{1}\right)$ and $\left(b_{2}\right)$ have the following forms (cf. Apendix A).

\section{$\underline{\text { Problem } \underline{b}_{1}}$}

$$
h_{1}=h-m_{\sigma} F_{T}>0 \text {. }
$$




\section{$\underline{\text { Problem } b_{2}}$}

$$
\begin{aligned}
& H=h+\boldsymbol{g}_{p}: \boldsymbol{M F}_{\boldsymbol{\sigma}}-\frac{1}{p}\left(m_{\sigma}+\bar{\gamma}_{\sigma} \xi \boldsymbol{g}_{p}: \boldsymbol{M F}_{\sigma}\right)\left(F_{T}-\gamma_{5} \boldsymbol{\alpha}: \boldsymbol{M \boldsymbol { F } _ { \sigma }}\right)>0 \\
& m_{\sigma}=\frac{1}{\rho_{0} c_{\sigma}}\left[\gamma_{0}\left(\boldsymbol{\sigma}: \boldsymbol{F}_{\sigma}-\Pi \cdot b\right)-\gamma_{3} T\left(\frac{\partial \Pi\left(Y_{K}^{T \sigma}\right)}{\partial T} \cdot b\right)\right] \\
& \xi=\frac{1-p}{M_{\alpha}^{2}}=\frac{T}{\rho_{0} c_{\sigma}}, \quad p=\frac{c_{\varepsilon}}{c_{\sigma}}, \quad M_{\alpha}^{2}=\boldsymbol{\alpha}: \boldsymbol{M} \boldsymbol{\alpha} .
\end{aligned}
$$

In the case of associated laws of plastic flow $\left(\boldsymbol{F}_{1, \boldsymbol{\sigma}}=\boldsymbol{F}_{\sigma}\right)$ the quantity $\left(m_{\sigma}=m\right)$ was analysed in (Mróz and Raniecki, [4], [5]; Śloderbach, [1], [3], [7], [30]; Śloderbach and Pajak, [28]) when all the elastic-plastic coupling effects being rejected $\left(\gamma_{9}=\bar{\gamma}_{9}=\gamma_{10}=\bar{\gamma}_{10}=\gamma_{11}=\bar{\gamma}_{11}=\gamma_{12}=\bar{\gamma}_{12}=0\right)$. By analysing the cyclic isothermal process in the space of stresses, the autor of those work observed that for the majority of materials (in particular for metallic materials) $m_{\sigma}$ is in general positive

$$
m_{\sigma}>0 \text {. }
$$

The inequalities (2.5) and (2.6) are a generalization of the uniqueness conditions derived by Mróz and Raniecki [4], [5], Raniecki, [6], Raniecki and Sawczuk, [35], [36]. This generalization consists in the non-associated laws of plastic flow being taken into account as well as the influence of plastic deformations on the thermoelastic properties of the body. The conditions obtained in the mentioned above papers can also be obtained from Eqs (3.3) and (3.4) by rejecting all the effects of elastic-plastic coupling $\left(\gamma_{9}=\bar{\gamma}_{9}=\gamma_{10}=\bar{\gamma}_{10}=\gamma_{11}=\bar{\gamma}_{11}=\gamma_{12}=\bar{\gamma}_{12}=0\right)$ and assuming associated laws of plastic flow $\left(\boldsymbol{F}_{1, \mathrm{\sigma}}=\boldsymbol{F}_{\mathrm{\sigma}}\right)$.

Condition (2.6) should be understood as a limitation for the functions occurring in the group of constitutive equations. If $(H=0)$, then from the theory of thermo-elasto-plasticity it results that an instantaneous change of stresses and temperature is possible when the body element is not being deformed ( $\dot{\boldsymbol{\varepsilon}}=\boldsymbol{0})$ and it does not exchange heat with the environment $\left(q_{0}=0\right)$. It means theoretically the possibility that if $\left(H=0\right.$ and $\dot{\boldsymbol{\varepsilon}}=\boldsymbol{0}, q_{0}=0$, then $\dot{\boldsymbol{\sigma}} \neq \boldsymbol{0}$ and $\left.\dot{\boldsymbol{T}} \neq 0\right)$. However, such phenomena do not take place in real physical bodies.

If $\left(h_{1}=0\right)$, then in an adiabatic process $\left(q_{0}=0\right)$ the body behaves similarly as the perfectly plastic body (no hardening), i.e., temporary adiabatic flow $(\dot{\boldsymbol{\varepsilon}} \neq \boldsymbol{0})$ is possible under constant stresses $(\dot{\boldsymbol{\sigma}}=\boldsymbol{0})$. Such a phenomenon can occur in the range of large deformations, so we assume that constitutive functions satisfy condition (2.5).

It is worthwhile to observe that in the case of metallic materials the satisfaction of condition (2.5) implies, in general, satisfaction of condition (2.6) [cf. (Mróz and Raniecki [4], [5]; Śloderbach [1], [3], [7], [30]; Śloderbach and Pajak [28])].

Let us assume that conditions (2.5) and (2.6) are both satisfied. The solutions of the incremental problems $\left(b_{1}\right)$ and $\left(b_{2}\right)$ can be expressed in the following forms

\section{$\underline{\text { Problem } b_{1}}$}

$$
\begin{aligned}
& \dot{\boldsymbol{\varepsilon}}=\boldsymbol{L}^{\boldsymbol{a}} \dot{\boldsymbol{\sigma}}+\frac{j}{h_{1}} \overline{\boldsymbol{K}} \dot{\boldsymbol{\sigma}}+\frac{j}{h_{1}} q F_{T}\left[\left(\boldsymbol{F}_{1, \boldsymbol{\sigma}}+\bar{\gamma}_{9} \boldsymbol{Z} * b\right)+\gamma_{4} m_{\sigma} \boldsymbol{\alpha}\right]-\gamma_{4} q \boldsymbol{\alpha}, \\
& \dot{T}=-\bar{\gamma}_{7} \xi \boldsymbol{\alpha}: \dot{\boldsymbol{\sigma}}+\frac{j m_{\sigma}}{h_{l}}\left[\left(\boldsymbol{F}_{\boldsymbol{\sigma}}-\bar{\gamma}_{7} \xi F_{T} \boldsymbol{\alpha}\right): \dot{\boldsymbol{\sigma}}-F_{T} q\right]+q,
\end{aligned}
$$




$$
\begin{aligned}
& \dot{\boldsymbol{\varepsilon}}^{p}=\frac{j}{h_{l}}\left[\left(\boldsymbol{F}_{\boldsymbol{\sigma}}-\bar{\gamma}_{7} \xi F_{T} \boldsymbol{\alpha}\right): \dot{\sigma}-F_{T} q\right] \boldsymbol{F}_{\boldsymbol{\sigma}}, \\
& \dot{\boldsymbol{\varepsilon}}^{e}=\boldsymbol{L}: \dot{\boldsymbol{\sigma}}+\gamma_{4} \dot{T} \boldsymbol{\alpha}+\frac{j}{h_{l}}\left[\left(\boldsymbol{F}_{\boldsymbol{\sigma}}-\bar{\gamma}_{7} \xi F_{T} \boldsymbol{\alpha}\right): \dot{\sigma}-F_{T} q\right]\left(\bar{\gamma}_{9} \boldsymbol{Z} * b\right)
\end{aligned}
$$

where

$$
\begin{aligned}
& \overline{\boldsymbol{K}}=\left[\left(\boldsymbol{F}_{1, \boldsymbol{\sigma}}+\bar{\gamma}_{9} \boldsymbol{Z} * b\right)+\gamma_{4} m_{\sigma} \boldsymbol{\alpha}\right] \otimes\left(\boldsymbol{F}_{\boldsymbol{\sigma}}-\bar{\gamma}_{7} \xi F_{T} \boldsymbol{\alpha}\right), \\
& \boldsymbol{L}^{\boldsymbol{a}}=\boldsymbol{L}-\gamma_{4} \bar{\gamma}_{7} \xi(\boldsymbol{\alpha} \otimes \boldsymbol{\alpha}), \quad q=\frac{1}{\rho_{0} c_{\sigma}} \operatorname{div} \boldsymbol{q}, \\
& j=\left\{\begin{array}{l}
1 \text { if } F=0 \text { and }\left(\boldsymbol{F}_{\boldsymbol{\sigma}}-\bar{\gamma}_{7} \xi F_{T} \boldsymbol{\alpha}\right): \dot{\sigma}-F_{T} q \geq 0, \\
0 \text { if } F=0 \text { or } F=0 \text { and }\left(\boldsymbol{F}_{\boldsymbol{\sigma}}-\bar{\gamma}_{7} \xi F_{T} \boldsymbol{\alpha}\right): \dot{\sigma}-F_{T} q<0,
\end{array}\right. \\
& \Lambda=\frac{j}{h_{l}}\left[\left(\boldsymbol{F}_{\boldsymbol{\sigma}}-\bar{\gamma}_{7} \xi F_{T} \boldsymbol{\alpha}\right): \dot{\sigma}-F_{T} q\right] .
\end{aligned}
$$

The symbol $\boldsymbol{L}^{a}$ denotes the tensor of adiabatic elasticity. Let us observe that the second right-hand term of Eq.(2.10) is not equal to the plastic strain rate, but may be considered as representing the adiabatic plastic strain rate. The tensor $\overline{\boldsymbol{K}}$ is asymmetric, $\bar{K}_{i j m n} \neq \bar{K}_{m n i j}$. A lack of symmetry is caused by not only thermal expansion accompanying power dissipation of the plastic strain and a change of the yield point together with an increase of temperature resulting from the piezoelectric effect, but also effects of elasticplastic coupling and the assumption of non-associated laws of plastic flow. It makes difficult a proof of the theorem concerning the uniqueness of solution of the incremental problem in the case of heterogeneous processes and in a consequence, the formulation of suitable criteria of bifurcation. The equations for the thermodynamic flow rate can also be expressed in terms of $\dot{\sigma}$ and $q$, They have the form

$$
\dot{K}=\frac{j}{h_{l}}\left[\left(\boldsymbol{F}_{\sigma}-\bar{\gamma}_{7} \xi F_{T} \boldsymbol{\alpha}\right): \dot{\sigma}-F_{T} q\right] b\left(X^{d}, Y_{K}^{T}\right)
$$

Taking into considerations the Gyarmati postulate (Gyarmati, [9]) and the resulting condition (cf. Śloderbach, [1], [3], [7], [30]) relation (2.13) takes the form

$$
-\dot{K}=\frac{j}{h_{l}}\left[\left(\boldsymbol{F}_{\sigma}-\bar{\gamma}_{7} \xi F_{T} \boldsymbol{\alpha}\right): \dot{\sigma}-F_{T} q\right] \frac{\partial F_{1}\left(X^{d}, Y_{K}^{T}\right)}{\partial \Pi} .
$$

\section{$\underline{\text { Problem } \underline{b}_{2}}$}

The alternative constitutive equations corresponding to equations, (cf. Śloderbach, [1], [3], [7], [30]) have the forms

$$
\dot{\sigma}=\boldsymbol{M}^{\boldsymbol{a}} \dot{\boldsymbol{\varepsilon}}+\frac{\gamma_{4} q}{p} \boldsymbol{M} \boldsymbol{\alpha}-\frac{j_{1}}{H} \tilde{\boldsymbol{K}} \dot{\boldsymbol{\varepsilon}}-\frac{j_{1} q}{p H}\left(\gamma_{5} \boldsymbol{\alpha}: \boldsymbol{M F}_{\boldsymbol{\sigma}}-F_{T}\right)\left(\tilde{\varphi} \boldsymbol{M} \boldsymbol{\alpha}+\boldsymbol{B}_{\boldsymbol{N}}\right)
$$




$$
\begin{aligned}
& \dot{T}=\frac{1}{p}\left(\bar{\gamma}_{7} \xi \boldsymbol{\alpha}: \boldsymbol{M F}_{\boldsymbol{\sigma}}+q\right)+\frac{j_{1}}{p H}\left(m_{\sigma}+\bar{\gamma}_{6} \xi \boldsymbol{g}_{p}: \boldsymbol{M \boldsymbol { F } _ { \sigma }}\right)\left[\boldsymbol{B}: \dot{\boldsymbol{\varepsilon}}+\frac{q}{p}\left(\gamma_{5} \boldsymbol{\alpha}: \boldsymbol{M \boldsymbol { F } _ { \sigma }}-F_{T}\right)\right], \\
& \dot{\boldsymbol{\varepsilon}}^{p}=\frac{j}{H}\left[\boldsymbol{B}: \dot{\boldsymbol{\varepsilon}}+\frac{q}{p}\left(\gamma_{5} \boldsymbol{\alpha}: \boldsymbol{M} \boldsymbol{F}_{\sigma}-F_{T}\right)\right] \boldsymbol{F}_{l, \boldsymbol{\sigma}}
\end{aligned}
$$

where

$$
j_{1}=\left\{\begin{array}{l}
1 \quad \text { if } \quad F=0 \text { and } \boldsymbol{B}: \dot{\boldsymbol{\varepsilon}}+\frac{q}{p}\left(\gamma_{5} \boldsymbol{\alpha}: \boldsymbol{M \boldsymbol { F } _ { \boldsymbol { \sigma } }}-F_{T}\right) \geq 0, \\
0 \quad \text { if } F<0 \text { or } F=0 \text { and } \boldsymbol{B}: \dot{\boldsymbol{\varepsilon}}+\frac{q}{p}\left(\gamma_{5} \boldsymbol{\alpha}: \boldsymbol{M} \boldsymbol{F}_{\boldsymbol{\sigma}}-F_{T}\right)<0
\end{array}\right.
$$

and

$$
\boldsymbol{M}^{\boldsymbol{a}}=\boldsymbol{M}+\frac{\gamma_{5} \bar{\gamma}_{6}}{p} \xi(\boldsymbol{M} \boldsymbol{\alpha}) \otimes(\boldsymbol{M} \boldsymbol{\alpha}), \quad \boldsymbol{B}_{\boldsymbol{N}}=\boldsymbol{B}-\boldsymbol{N}_{\boldsymbol{Z}} \quad-\boldsymbol{L} \boldsymbol{N}=-\boldsymbol{L}\left(Y_{K}^{T \sigma}\right) \boldsymbol{N}\left(Y_{K}^{T \varepsilon}\right)=\boldsymbol{Z},
$$

where $\quad \boldsymbol{L}\left(Y_{K}^{T \sigma}\right)=\frac{\partial \varepsilon^{e}\left(Y_{K}^{T \sigma}\right)}{\partial \sigma}, \quad \boldsymbol{N}\left(Y_{K}^{T \varepsilon}\right)=\frac{\partial \sigma\left(Y_{K}^{T \varepsilon}\right)}{\partial K}, \quad \boldsymbol{Z}\left(Y_{K}^{T \sigma}\right)=\frac{\partial \boldsymbol{\varepsilon}^{e}\left(Y_{K}^{T \sigma}\right)}{\partial K}$,

and

$$
\boldsymbol{B}=\boldsymbol{M} \boldsymbol{F}_{\sigma}+\bar{\gamma}_{6} \frac{\xi}{p}\left(\gamma_{5} \boldsymbol{\alpha}: \boldsymbol{M F}_{\sigma}-F_{T}\right), \quad \tilde{\boldsymbol{K}}=\left[\tilde{\varphi} \boldsymbol{M} \boldsymbol{\alpha}-\boldsymbol{N}_{\boldsymbol{Z}}\right] \otimes \boldsymbol{B}+\boldsymbol{B} \otimes \boldsymbol{B}
$$

The following additional quantities are involved in the tensor $\tilde{\boldsymbol{K}}$

$$
\begin{aligned}
& \tilde{\varphi}=\gamma_{5} \frac{m_{\sigma}}{p}+\bar{\gamma}_{6} \frac{\xi}{p} F_{T}-\bar{\gamma}_{6} \bar{\gamma}_{10} \frac{\xi}{p}\left\{\alpha:\left[\boldsymbol{N} *\left(\gamma_{3} b+\gamma_{5} F_{1, \Pi}\right)\right]\right\}, \\
& \boldsymbol{N}_{\boldsymbol{Z}}=\bar{\gamma}_{10} \boldsymbol{N} * b+\gamma_{12} \boldsymbol{N} * F_{1, \Pi}=\boldsymbol{N} *\left(\bar{\gamma}_{10} b+\gamma_{12} F_{1, \Pi}\right),
\end{aligned}
$$

where

$$
F_{1, \Pi}=\frac{\partial F}{\partial \Pi}
$$

The symbol $\boldsymbol{M}^{\boldsymbol{a}}$ denotes the tensor of adiabatic moduli of elasticity. Similarly to the former case, the tensor interrelating the stress rate and strain rate is asymmetric because $\tilde{K}_{i j m n} \neq \tilde{K}_{m n i j}$.

Let us observe that, if conditions (2.5) and (2.6) are both satisfied, Eqs (2.15) are equivalent Eqs (2.10). They can be obtained by solving Eq. $(2.10)_{1}$ for $\dot{\sigma}$ and substituting the result into Eqs $(2.10)_{2}$ and (2.10) $)_{3}$. Equations (2.10) can be obtained by solving Eq.(2.15) $)_{1}$ for $\dot{\boldsymbol{\varepsilon}}$ and substituting the result into Eqs $(2.15)_{2}$ and $(2.15)_{3}$. Thus, conditions (2.5) and (2.6) are often called conditions of reciprocal inversability of constitutive equations in relation to $\dot{\varepsilon}$ and $\dot{\sigma}$.

If all the thermodynamic coupling effects in Eqs (2.10) - (2.14) and (2.15) - (2.16) are rejected $\left(\gamma_{1}=\gamma_{3}=\bar{\gamma}_{6}=\bar{\gamma}_{7}=\bar{\gamma}_{9}=\bar{\gamma}_{10}=\gamma_{12}=0\right)$ and if $\left(\boldsymbol{F}_{1, \sigma}=\boldsymbol{F}_{\sigma}\right)$, those equations will constitute two equivalent sets of fundamental equations of the theory of thermal stresses in an elastic-plastic body. Then $(q=\dot{T})$ or ( $\left.-\operatorname{div} \mathbf{q}=\rho_{0} c_{\sigma} \dot{T}\right)$ and $(p=1)$. 


\section{Formulation of the incremental boundary value problem}

If condition (2.5) is satisfied, the set of Eqs (2.10) - (2.14) is equivalent to the fundamental set of Eqs (2.15) - (2.16) together with the relevant evolution equations of internal parameters $\dot{K}$, when $H>0$. The set of those equations, together with the law of heat conduction, with the equation of motion and the kinematic relations

$$
\operatorname{div} \dot{\sigma}+\rho_{0} \dot{\boldsymbol{b}}_{m}=\rho_{0} \dot{\boldsymbol{v}} \quad \text { and } \quad 2 \dot{\varepsilon}_{i j}=v_{i, j}+v_{j, i}
$$

where $\boldsymbol{v}$ - is the vector of velocity of particles, $\boldsymbol{b}_{m}-$ is the body force, constitute a set of fundamental field equations of generalized coupled thermo-elasic-plasticity. Together with the boundary conditions and the initial conditions it may be used as a basis for the analysis of many problems of generalized coupled thermoelasic-plasticity, both dynamic and static (cf. Śloderbach, [1], [2], [7], [30]).

The following static incremental boundary-value problems can be formulated (cf. Raniecki, [6]; Raniecki and Sawczuk, [36]; Śloderbach, [1], [3], [7], [30]).

Let the body occupy, at a time $t_{0}$, a region $D$ in space. Let us denote by $\bar{D}$ the closure of $D$ and by the symbol $S$ - the boundary of $\bar{D} . S$ is the closure of the sum of non-intersecting regular open surfaces $S_{v}$ and $S_{t}$. Let the thermodynamic state of the body

$$
\{T, \sigma, K\}
$$

and the rate of body forces $\dot{\boldsymbol{b}}_{m}$ be known, at a time $t_{0}$ and at every point $\boldsymbol{x}$ of the closure $\bar{D}$. It is assumed that the functions (3.2) satisfy the condition $F \leq 0$. It is also assumed that the values of the surface forces $\dot{\boldsymbol{t}}_{0}$ and the velocities of material points $\boldsymbol{v}^{0}$ are known at time $t_{0}$ over the parts $S_{v}$ and $S_{t}$ of the boundary, that is

$$
\left\{\begin{array}{l}
\dot{\boldsymbol{\sigma}} \boldsymbol{n}=\dot{\boldsymbol{t}}_{0} \quad \text { for } \quad \boldsymbol{x} \in S_{t}, \\
\boldsymbol{v}=\boldsymbol{v}_{0} \quad \text { for } \quad \boldsymbol{x} \in S_{v}
\end{array}\right.
$$

where $\boldsymbol{n}$ - is a unit vector normal to $S$, directed towards the outside of $D$. Our task is to find the set of functions $(\dot{\boldsymbol{\sigma}}, \dot{\boldsymbol{\varepsilon}}, \boldsymbol{v})$ defined in $\bar{D}$ and the function $\dot{T}$ defined in $D$, which satisfy, in the region $D$, Eqs (2.3), (2.6), (3.1), the expression $q=\frac{1}{\rho_{0} c_{\sigma}} \operatorname{divq}$ and the incremental equations of equlibrium

$$
\operatorname{div} \dot{\sigma}+\rho_{0} \dot{\boldsymbol{b}}_{m}=0
$$

Let us observe that, knowing the functions (3.2), we can determine $q$ at every point of the region $D$, directly from the $\boldsymbol{q}=\boldsymbol{\varphi}^{q}\left(\nabla T, Y_{K}^{T}\right)$, where $\nabla T=\operatorname{grad} T$ and $Y_{K}^{T}=\{T, K\}$, by differentiating $T$ and $\boldsymbol{q}$ with respect to the coordinate variables $x_{i}$, where $i=1,2,3$.

In the coupled generalized thermo-elasto-plasticity the formulated incremental boundary problem plays the same role as a suitable boundary incremental problem in the isothermal theory of plasticity. Namely, if its solution is ambiguous, then a solution of a general problem, where the history of variation of surface forces, velocity and temperature on the surface of the body under consideration are given, is ambiguous, too (Raniecki,[6]; Śloderbach, [1], [3], [7], [30]). 


\section{Discussion of uniqueness conditions}

Tests of uniqueness of the solution of the incremental boundary problem presented in Section 3 belong to the most important problems shown in this paper. Such tests and the results obtained can be the basis for the formulation of two criteria allowing the estimation of the critical thermodynamic state after exceeding of which bifurcation of the equilibrium state is possible. These criteria are also two sufficient conditions of the uniqueness of solution of the incremental boundary problem, called local sufficient condition and global sufficient condition.

The local condition can be easily applied in practice because it is directly expressed by constitutive functions and material constants. However, it gives less accurate estimations of the critical state. The global condition gives better estimations of critical states but its application is more difficult because it requires searching kinematically acceptable velocity fields for which the functional $J$ (see subsection 4.4 ) reaches zero. Derivation of both conditions uses methods presented in the previous papers (Mróz and Raniecki, [4], [5]; Raniecki, [6], [33]; Raniecki and Bruhns, [34]; Śloderbach, [1], [3], [7], [30]).

\subsection{Local uniqueness condition}

The following theorem is proved in Appendix B of the paper.

\section{Theorem 1.}

If the inequality

$$
h_{l}=h-m_{\sigma} F_{T}>\frac{1}{2}\left[\sqrt{\left(\boldsymbol{g}: \boldsymbol{M}^{\boldsymbol{a}} \boldsymbol{g}\right)\left(\overline{\boldsymbol{F}}_{\boldsymbol{\sigma}}: \boldsymbol{M}^{\boldsymbol{a}} \overline{\boldsymbol{F}}_{\boldsymbol{\sigma}}\right)}-\boldsymbol{g}: \boldsymbol{M}^{\boldsymbol{a}} \overline{\boldsymbol{F}}_{\boldsymbol{\sigma}}\right]=h_{l}^{*}
$$

where

$$
\boldsymbol{g}=\left(F_{1, \boldsymbol{\sigma}}+\bar{\gamma}_{9} \boldsymbol{Z} * b+\gamma_{4} m_{\sigma} \boldsymbol{\alpha}\right) \quad \text { and } \quad \overline{\boldsymbol{F}}_{\boldsymbol{\sigma}}=\left(\boldsymbol{F}_{\boldsymbol{\sigma}}-\bar{\gamma}_{7} \xi F_{T} \boldsymbol{\alpha}\right),
$$

is satisfied at every point of the plastic portion of the body $D_{p}=\{\boldsymbol{x}: F=0\}$, there can exist only one set of functions $\{\dot{\sigma}, \dot{\varepsilon}, \dot{T}\}$ of class $C^{l}$ at least, which is a solution of the incremental boundary value problem of generalized coupled thermoplasticity, which was formulated in section 3 .

Inequality (4.1) is the sufficient local uniqueness condition. Each thermodynamic state, for which condition (4.1) is satisfied, is secure against bifurcation. Since in the course of a deformation process of the body the value of the strain-hardening function (the modulus) decreases, in general, therefore the value of $h_{l}^{*}$ may be treated as an upper estimation of the unknown critical value $h$ corresponding to the critical state.

Some particular cases of expression (4.1) have already been mentioned in the literature (Śloderbach, [1], [7], [30]; Śloderbach and Pajak, [28]). A similar condition was obtained by Mróz in Mróz [37], [38] and Hueckel and Maier [39], [40] in their analysis of the stability of material defined as a condition of half the product of the stress rate tensor and the strain rate tensor being positive. Their study was confined to the case of the isothermal theory of plasticity (with no thermo-mechanical couplings), the elastic plastic coupling effects and non-associated laws of plastic flow being preserved. An expression of this type was also obtained in Raniecki [6], [33], Raniecki and Bruhns [34], Śloderbach [1], [3], [7], [30], Śloderbach and Pajak, [28] for the case of isothermal uncoupled and non-isothermal coupled theory of thermoplasticity with a nonassociated law of plastic flow. 


\subsection{The global uniqueness condition}

\subsubsection{Global condition for a thermo-elasto-plastic body dependent on kinematically admissible strain rates fields}

Let us assume that there exist two sets of functions $\{\dot{\boldsymbol{\sigma}}, \dot{\boldsymbol{\varepsilon}}, \dot{T}, \boldsymbol{v}\}$ and $\left\{\dot{\boldsymbol{\sigma}}^{*}, \dot{\boldsymbol{\varepsilon}}^{*}, \dot{T}^{*}, \boldsymbol{v}^{*}\right\}$ which are solutions of the incremental boundary-value problem of generalized coupled thermoplasticity, which was formulated in section 3 . Then, the following equality must be satisfied

$$
\Lambda^{*}=\int_{D}\left(\dot{\sigma}-\dot{\sigma}^{*}\right):\left(\dot{\boldsymbol{\varepsilon}}-\dot{\boldsymbol{\varepsilon}}^{*}\right) d V=0
$$

due to the fact that both solutions satisfy the same boundary conditions (2.5), in the case of Gauss Ostrogradski theorem.

Let us denote by $J$ the integrand in expression (4.3), which depends on $\left(\dot{\varepsilon}\right.$ and $\left.\dot{\varepsilon}^{*}\right)$, for an elasticplastic body, as follows

$$
J\left(\dot{\varepsilon}, \dot{\varepsilon}^{*}, j_{l}, j_{l}^{*}\right)=\left[\dot{\sigma}(\dot{\varepsilon})-\dot{\sigma}\left(\dot{\varepsilon}^{*}\right)\right]: \Delta \dot{\varepsilon}
$$

where $\Delta \dot{\boldsymbol{\varepsilon}}=\dot{\boldsymbol{\varepsilon}}-\dot{\boldsymbol{\varepsilon}}^{*}$ and $\dot{\sigma}^{*}=\dot{\sigma}\left(\dot{\varepsilon}^{*}\right)$ and $j_{1}=j_{1}(\dot{\boldsymbol{\varepsilon}})$ and $j_{l}^{*}=j_{l}\left(\dot{\varepsilon}^{*}\right)$ are defined by Eqs $(2.16)_{1,2}$. Functions $j$ and $j^{*}$ take the value 1 - for the active plastic deformation, or $0-$ for the elastic loading or plastic unloading, see Eqs (2.16) $)_{1,2}$ and Śloderbach [1], [3], [7], [30].

The quantities ( $\dot{\varepsilon}$ and $\left.\dot{\varepsilon}^{*}\right)$ and $\left(\dot{\sigma}^{*}\right.$ and $\left.\dot{\varepsilon}^{*}\right)$ are interrelated by Eq. $(2.15)_{1}$, which can be rewritten in a more compact form as follows

$$
\dot{\sigma}=M_{l} \dot{\varepsilon}-M_{l} \boldsymbol{d}_{1}-\frac{j_{1}}{H_{1}} \boldsymbol{g}^{*}\left[\overline{\boldsymbol{F}}_{\sigma}^{*}:\left(\dot{\varepsilon}-\boldsymbol{d}_{1}\right)+Z_{1}\right]
$$

where

$$
\left\{\begin{array}{l}
\boldsymbol{g}^{*} \equiv \boldsymbol{M}_{1} \boldsymbol{g}=\tilde{\varphi}(\boldsymbol{M} \boldsymbol{\alpha})-\boldsymbol{N}_{\boldsymbol{Z}}+\boldsymbol{B}, \quad \text { and } \quad \overline{\boldsymbol{F}}_{\boldsymbol{\sigma}}^{*} \equiv \boldsymbol{M}_{1} \overline{\boldsymbol{F}}_{\boldsymbol{\sigma}}=\boldsymbol{B} \\
\boldsymbol{d}_{1}=\gamma_{5} q \boldsymbol{\alpha}, \quad \boldsymbol{M}_{1} \equiv \boldsymbol{M}^{a}, \quad \boldsymbol{Z}_{1} \equiv-q F_{T}, \quad H_{1} \equiv H .
\end{array}\right.
$$

Since expression (4.3) with zero at the right side provides existence of two sets of functions $\{\dot{\boldsymbol{\sigma}}, \dot{\varepsilon}, \dot{T}, \boldsymbol{v}\}$ and $\left\{\dot{\boldsymbol{\sigma}}^{*}, \dot{\boldsymbol{\varepsilon}}^{*}, \dot{T}^{*}, \boldsymbol{v}^{*}\right\}$, which are a solution of the formulated incremental boundary problem, so positivity of expression (4.3), i.e., $\Lambda^{*}>0$ (Raniecki, [6], [33]; Raniecki and Bruhns, [34]; Raniecki and Sawczuk, [36]; Śloderbach, [1], [3], [7], [30]; Śloderbach and Pajak, [28]) and Eq.(4.4) will be a condition excluding the occurrence of the bifurcation state. Inequality $\Lambda^{*}>0$, is a sufficient global condition of uniqueness and a global criterion excluding the occurrence of the bifurcation state.

\subsubsection{Global condition for a comparison body dependent on kinematically admissible strain velocity fields}

Let us introduce the following function $J$, depending on $\left(\dot{\varepsilon}\right.$ and $\left.\dot{\varepsilon}^{*}\right)$

$$
J^{\prime}\left(\dot{\boldsymbol{\varepsilon}}, \dot{\boldsymbol{\varepsilon}}^{*}\right)=\Delta \dot{\boldsymbol{\varepsilon}}: \boldsymbol{M}_{1} \Delta \dot{\boldsymbol{\varepsilon}}-\frac{1}{4 x^{2} H}\left[\left(\boldsymbol{g}^{*}+x^{2} \overline{\boldsymbol{F}}_{\boldsymbol{\sigma}}^{*}\right): \Delta \dot{\boldsymbol{\varepsilon}}\right]^{2}
$$


where $x^{2}-$ is a scalar quantity.

The expression $J$ is a comparison body function and represents a one-parameter family of expressions of $J$, with respect to the parameter $x^{2}$.

The idea of reference body in coupled thermoplasticity was introduced in the papers by Mróz and Raniecki [4], [5], Raniecki [6], [33], Raniecki and Brunhs [34] and also in the author's papers (Śloderbach, [1], [3], [7], [30]; Śloderbach and Pajak, [28]).

The functions $J$ and $J$ depend in addition to the variables ( $\dot{\varepsilon}$ and $\dot{\varepsilon}^{*}$ ) on the thermodynamic state of the body (2.5). As it results from a comparison of expression (4.7) and (4.4), it presents a certain linear dependence between $(\Delta \dot{\sigma}$ and $\Delta \dot{\varepsilon})$. Differentiating $J^{\prime}$ in relation to $\Delta \dot{\varepsilon}$ we obtain a linear dependence between $\Delta \dot{\sigma}$ and $\Delta \dot{\varepsilon}$, which does not occur in expression (4.4), because these dependences are nonlinear.

\section{Lemma 1}

It will be shown that if the same thermodynamic state is prescribed for $J$ and $J^{\prime}$, then for each pair ( $\dot{\varepsilon}$ and $\dot{\varepsilon}^{*}$ ) the following inequality holds

$$
J\left(\dot{\varepsilon}, \dot{\varepsilon}^{*}, j_{l}, j_{l}^{*}\right)-J^{\prime}\left(\dot{\varepsilon}, \dot{\varepsilon}^{*}\right) \geq 0
$$

The proof of lemma 1 is given in the works of the author (Śloderbach, [1], [3], [7], [30]).

Using expression (4.3), inequalitie (4.8) we can formulate the following sufficient condition of uniqueness of a solution of the incremental boundary problem for the comparison body which is a stronger (safer) criterion excluding the occurrence of the bifurcation state.

\section{Theorem 2}

Let us now formulate a sufficient global uniqueness criterion (that is a criterion which excludes bifurcation). Let $H>0$ at every point $\boldsymbol{x} \in D_{p}$, in this part, where plastic deformations are occurring, i.e., where $D_{p}=\{\boldsymbol{x}: F=0\}$. If for every non-zero kinematically admissible and integrable velocity field $\boldsymbol{v}$, which vanishes over the part $S_{v}$ of the surface, the inequality

$$
\int_{D} J_{1}^{\prime}(\boldsymbol{v}) d V-\int_{D_{p}} J_{2}^{\prime}(\boldsymbol{v}) d V>0,
$$

is satisfied, there exists only one pair $\{\dot{\sigma}, \dot{T}\}$ constituting a solution of the incremental boundary-value problem in generalized coupled thermoplasticity. This criterion can easily be demonstrated.

\section{$\underline{\text { Proof }}$}

The integrands in Eqs (4.9) are

$$
J_{1}^{\prime}(\dot{\boldsymbol{\varepsilon}})=\dot{\boldsymbol{\varepsilon}}: \boldsymbol{M}_{1} \dot{\boldsymbol{\varepsilon}} \quad \text { and } \quad J_{2}^{\prime}(\dot{\boldsymbol{\varepsilon}})=\frac{1}{4 x^{2} H}\left[\left(\boldsymbol{g}^{*}+x^{2} \overline{\boldsymbol{F}}_{\boldsymbol{\sigma}}^{*}\right): \dot{\boldsymbol{\varepsilon}}\right] .
$$

The validity of the sufficient global uniqueness criterion (4.9), being a safer criterion excluding the state of bifurcation, follows directly from the inequalities $\Lambda^{*}>0$ and Eq.(4.8), see comments in Śloderbach [1], [3], [7], [30]. 
The integral condition (4.9) is, in particular form, of essential practical importance. If, for a prescribed state $\{T, \sigma, K\}$ it is impossible to find such a field $\boldsymbol{v}$ that the sum of integrals at the left-hand side of the expression (4.9) is zero, we are assured that this state is secure against bifurcation.

The idea of deriving such a criterion for the incremental boundary-value problem for coupled thermoplasticity in the case of associated laws of plastic flow and for small deformations has been derived by Mróz and Raniecki [4], [5], Raniecki [6], and for the case of non-associated laws of plastic flow by Śloderbach [1], [3], [7], [30] and for large deformations by Raniecki [33], Raniecki and Bruhns [34] Śloderbach and Pająk [28]. Another sufficient global uniqueness criterion for incremental problems of isothermal plasticity of elastic-plastic bodies with non-associated laws of plastic flow has been given by Huecel and Maier [39], [40], Maier [41].

It will be shown in Appendix $\mathrm{C}$ that the sufficient local uniqueness condition following from the requirement that the intergrand $J$ ' should be definite positive is the same as for a generalized thermo-elasticplastic body Eqs $(2.12)_{2}$ and $(2.15)_{1}$ or (4.5) provided that the parameter $x^{2}$ takes its optimum form

$$
x_{0}^{2}=\left(\frac{\boldsymbol{g}^{*}: \boldsymbol{L}_{\mathbf{l}} \boldsymbol{g}^{*}}{\overline{\boldsymbol{F}}_{\sigma}^{*}: \boldsymbol{L}_{\boldsymbol{l}} \overline{\boldsymbol{F}}_{\sigma}^{*}}\right)^{\frac{1}{2}} .
$$

A procedure for obtaining the optimum parameter $x_{0}^{2}$ is also discussed in Appendix C.

For the parameter $x_{0}^{2}$ the sufficient local uniqueness condition becomes the optimum (strongest) condition for the entire one-parameter family of sufficient uniqueness conditions.

\subsubsection{Global condition for a thermo-elastic-plastic body depending on statically admissible stress rate fields}

Now, let the symbol $I$ mean the integrand from expression (4.3) dependent on ( $\dot{\sigma}$ and $\dot{\sigma}^{*}$ ) for a thermo-elastic-plastic body in the following way

$$
I\left(\dot{\sigma}, \dot{\sigma}^{*}, j, j^{*}\right)=\left[\dot{\varepsilon}(\dot{\sigma})-\dot{\varepsilon}\left(\dot{\sigma}^{*}\right)\right]: \Delta \dot{\sigma}
$$

where $\quad \Delta \dot{\boldsymbol{\sigma}}=\dot{\boldsymbol{\sigma}}-\dot{\boldsymbol{\sigma}}^{*}, \quad \dot{\boldsymbol{\sigma}}^{*}=\dot{\boldsymbol{\sigma}}\left(\dot{\varepsilon}^{*}\right) \quad$ and $\quad j=j(\dot{\boldsymbol{\sigma}})$ and $j^{*}=j\left(\dot{\boldsymbol{\sigma}}^{*}\right)$.

Like in subsection 4.2.1, functions $j$ and $j^{*}$ take the value $1-$ for the active plastic deformation, or 0 - for the elastic loading or plastic unloading, see (Śloderbach, [7], [30]).

At present $\dot{\sigma}^{*}$ and $\dot{\varepsilon}^{*}$ are connected with a suitable constitutive equation, see (Raniecki, [6]; Śloderbach, [1], [3], [7], [30]; Śloderbach and Pajak, [28]) written as

$$
\dot{\boldsymbol{\varepsilon}}=\boldsymbol{L}_{l} \dot{\sigma}+\frac{j}{h_{l}} \boldsymbol{g}\left[\overline{\boldsymbol{F}}_{\sigma} \dot{\sigma}+z_{l}\right]+\boldsymbol{d}_{1}
$$

where

$$
\left\{\begin{array}{l}
\boldsymbol{g}=\left(\boldsymbol{F}_{l, \boldsymbol{\sigma}}+\bar{\gamma}_{9} \xi \boldsymbol{Z} * b+\gamma_{4} m_{\sigma} \boldsymbol{\alpha}\right), \quad \boldsymbol{d}_{1}=\gamma_{4} q \boldsymbol{\alpha}, \\
\boldsymbol{Z}_{1}=-\boldsymbol{q} \boldsymbol{F}_{\boldsymbol{T}}, \quad \boldsymbol{L}_{l} \equiv \boldsymbol{L}^{\boldsymbol{a}}, \quad \boldsymbol{M}_{1} \equiv \boldsymbol{M}^{\boldsymbol{a}} .
\end{array}\right.
$$

Like in the case of kinematically admissible strain velocity field, expression (4.3) with the sign zero at the right side allows the existence of two sets of functions $\{\dot{\boldsymbol{\sigma}}, \dot{\boldsymbol{\varepsilon}}, \dot{T}, \boldsymbol{v}\}$ and $\left\{\dot{\sigma}^{*}, \dot{\boldsymbol{\varepsilon}}^{*}, \dot{T}^{*}, \boldsymbol{v}^{*}\right\}$, being a 
solution of the formulated incremental boundary-value problem. Thus, the positive definition of expression (4.3) is a condition excluding the occurrence of the bifurcation state, i.e., $\Lambda^{*}>0$ (Raniecki, [6], [33]; Raniecki and Bruhns, [34]; Śloderbach, [1], [3], [7], [30]; Śloderbach and Pajak, [28]). The positive definition of expression (4.12) is a result of the positive definition of (4.9), too. In this case, inequality (4.9) is a sufficient global condition of uniqueness of solution of the incremental boundary problem for a reference body and a global criterion excluding the occurrence of the bifurcation state for a case of kinematically admissible stress velocity fields, see comments in Śloderbach [1], [3], [7], [30].

\subsubsection{Global condition for a comparison body dependent on statically admissible stress rate fields}

The function $I^{\prime}$ dependent on $\dot{\sigma}$ and $\dot{\sigma}^{*}$ is introduced in the following way

$$
I\left(\dot{\boldsymbol{\sigma}}, \dot{\boldsymbol{\sigma}}^{*}\right)=\Delta \dot{\boldsymbol{\sigma}}: \boldsymbol{L}_{1} \Delta \dot{\boldsymbol{\sigma}}-\frac{1}{4 y^{2} h_{l}}\left[\left(\boldsymbol{g}-y^{2} \overline{\boldsymbol{F}}_{\boldsymbol{\sigma}}\right): \Delta \dot{\boldsymbol{\sigma}}\right]^{2}
$$

where $y^{2}$ is a scalar parameter.

The above expression expresses a one-parameter series of expressions $I$ ' related to the parameter $y^{2}$. The functions $I$ and $I^{\prime}$ depend not only on independent variables $\dot{\sigma}$ and $\dot{\sigma}^{*}$, but on the thermodynamic state as well (3.2).

In coupled generalized thermo-elasto-plasticity, an idea of the reference body dependent on statically acceptable stress velocity fields was introduced in the author's papers (Śloderbach, [7], [30]; Śloderbach and Pajak, [28], [29]). As in the case of kinematically admissible strain velocity fields, from a comparison of expression (4.15) with (4.12) it appears that it is a certain linear dependence between $(\Delta \dot{\sigma}$ and $\Delta \dot{\varepsilon})$. Differentiating $I^{\prime}$ in relation to $\Delta \dot{\sigma}$ we obtain a certain linear dependence between $\Delta \dot{\varepsilon}$ and $\Delta \dot{\sigma}$, which does not occur in expression (4.17), because in Eq. (4.12) those dependences are not linear.

\section{Lemma 2}

Let us demonstrate that under a given thermodynamic state, the same for $I$ and $I^{\prime}$, the following inequality is true for each pair $\left\{\dot{\sigma}\right.$ and $\left.\dot{\sigma}^{*}\right\}$ and each combination $j$ and $j^{*}$

$$
I\left(\dot{\sigma}, \dot{\sigma}^{*}, j, j^{*}\right)-I\left(\dot{\sigma}, \dot{\sigma}^{*}, j, j^{*}\right) \geq 0 .
$$

The proof of lemma 2 is given in (Śloderbach, [7], [30]).

Using the inequality $\Lambda^{*}>0$, see expression (4.3), and inequalities (4.16) we can formulate (like in the 4.2.2) the following sufficient condition of uniqueness of a solution of the incremenral boundary problem for the reference body dependent on statically admissible stress velocity fields, which is a safer criterion excluding the occurrence of the bifurcation state.

\section{$\underline{\text { Theorem } 3}$}

Let us assume $h_{1}>0$ at each point of the body $\boldsymbol{x} \in D_{P}$ in its part where plastic deformations take place, i.e., where $D_{P}=\{\boldsymbol{x}: F=0\}$. If for each statically admissible stress velocity field $\dot{\sigma}$ (or the field of stress velocity difference $\Delta \dot{\sigma}$ ), which disappears on a surface part (a body boundary) $S_{t}$, the following inequality is satisfied

$$
\int_{D} I^{\prime}{ }_{1}(\dot{\boldsymbol{\sigma}}) d V-\int_{D_{P}} I^{\prime}{ }_{2}(\dot{\boldsymbol{\sigma}}) d V>0,
$$


then only one pair $\{\dot{\boldsymbol{\varepsilon}}, \dot{T}\}$ being a solution of the incremental boundary problem of coupled generalized thermoplasticity can exist. It is easy to prove that the above introduced criterion is true, like in the case of kinematically acceptable strain velocity fields.

\section{$\underline{\text { Proof }}$}

Integrands occurring in expression (4.26) have the following form

$$
I_{l}^{\prime}(\dot{\boldsymbol{\sigma}})=\dot{\sigma}: \boldsymbol{L}_{l} \dot{\sigma} \quad \text { and } \quad I_{2}^{\prime}=-\frac{1}{4 y^{2} h_{l}}\left[\left(\boldsymbol{g}-y^{2} \overline{\boldsymbol{F}}_{\boldsymbol{\sigma}}\right): \dot{\sigma}\right]^{2}
$$

Truth of the above condition of uniqueness for a reference body being a safer criterion excluding bifurcation results directly from satisfying the inequality $\Lambda^{*}>0$, see expression (4.3) and inequalitie (4.16), see comments in Śloderbach, [7], [30], Śloderbach and Pajak [28].

Integral condition (4.17) presented in this form is very important from a practical point of view. Namely, if for a given thermodynamical state $\{T, \sigma, K\}$ it is not possible to find such statically admissible stress velocity field $\dot{\sigma}$, for which a sum of integrals occurring at the left side of the expression is equal to zero, then we must be sure that such a state is safe from the point of view of possibility of bifurcation state occurrence.

In Appendix D it is shown that the sufficient local condition of uniqueness resulting from the requirement of a positively defined integrand $I$ ' is the same as for the case of a thermo-elastic-plastic body $(2.10)_{1}$ and (4.13) when the parameter $y^{2}$ takes the following optimum form

$$
y_{0}^{2}=\left(\frac{\boldsymbol{g}: \boldsymbol{M}_{\mathbf{1}} \boldsymbol{g}}{\overline{\boldsymbol{F}}_{\boldsymbol{\sigma}}: \boldsymbol{M}_{\mathbf{1}} \overline{\boldsymbol{F}}_{\boldsymbol{\sigma}}}\right)^{\frac{1}{2}} .
$$

For the parameter $y_{0}^{2}$ the local condition of uniqueness becomes the optimum (safest) condition from all the sets of conditions.

Now we can state that in the case of the comparison body expressed by Eqs (4.7), (4.10) or as $J_{l}$ $(4.8)_{1}$ dependent on kinematically admissible strain velocity fields at the boundary transition $\left(\boldsymbol{g}^{*}=\overline{\boldsymbol{F}}_{\sigma}^{*}\right)$ and $\left(x^{2}=1\right)$, we obtain a body of coupled generalized thermoplasticity deteremined by Eq.(4.5). From expressions (4.15) or (4.18) for the reference body dependent on statically acceptable stress velocity fields it appears that substituting at the boundary $\left(\boldsymbol{g}=\overline{\boldsymbol{F}}_{\boldsymbol{\sigma}}\right)$ and the value $\left(y^{2}=1\right)$ we obtain the expression like for the thermoelastic body. Thus, reference bodies are not obtained by their mutual inversion like in the case of a thermo-elastic-plastic bodies. They are independently derived so as to satisfy inequalities (4.8), (4.9) for a reference body dependent on kinematically acceptable strain fields and inequalities (4.16), (4.17) for the reference body dependent on statically acceptable stress velocity fields, respectively.

\section{Appendices}

\subsection{Appendix A}

The procedure of derivation of necessary conditions of uniqueness (2.5) and (2.6) for the problems $\boldsymbol{b}_{\boldsymbol{1}}$ and $\boldsymbol{b}_{2}$ (see section 2) is similar as that from papers (Śloderbach, [1], [2], [30]; Raniecki, [4], [5]; Raniecki and Bruhns, [6]). 
1. To derive the condition $H>0$ (problem $\boldsymbol{b}_{2}$ ) let us assume first that for prescribed values of the strain rate $\dot{\varepsilon}$ and $\operatorname{div} \boldsymbol{q}$ the plastic loading process is active $\left(\dot{\boldsymbol{\varepsilon}}^{p} \neq \boldsymbol{0}\right)$, and let us denote by $\Lambda^{(p)}$ and $\dot{T}^{(p)}$ the corresponding values of $\Lambda$ and $\dot{T}$. Then, a set of algebraic equations can be obtained by substituting the expression on $\left(\dot{\boldsymbol{\varepsilon}}=\dot{\boldsymbol{\varepsilon}}^{e}+\dot{\boldsymbol{\varepsilon}}^{p}\right),(2.2)_{2},(2.15)_{1}$ and $(2.15)_{2}$, into association condition and Eq.(2.2) $)_{1}$. After transformation we can obtain

$$
\left\{\begin{array}{l}
\left(F_{T}-\gamma_{5} \boldsymbol{\alpha}: \boldsymbol{M F}_{\boldsymbol{\sigma}}\right) \dot{T}^{(p)}-\left(h+\boldsymbol{g}_{p}: \boldsymbol{M F}_{\boldsymbol{\sigma}}\right) \Lambda^{(p)}=-\boldsymbol{F}_{\boldsymbol{\sigma}}: \boldsymbol{M} \dot{\boldsymbol{\varepsilon}} \\
\dot{T}^{(p)}-\frac{1}{p}\left(m_{\varepsilon}+\bar{\gamma}_{6} \xi \boldsymbol{\alpha}: \boldsymbol{M F}_{\boldsymbol{\sigma}}\right) \Lambda^{(p)}=-\bar{\gamma}_{6} \frac{\xi}{p} \boldsymbol{\alpha}: \boldsymbol{M} \dot{\boldsymbol{\varepsilon}}-\frac{q}{p}
\end{array}\right.
$$

where

and

$$
\begin{aligned}
& m_{\varepsilon}=m_{\sigma}+\gamma_{3} \bar{\gamma}_{\sigma} \bar{\gamma}_{10} \xi \boldsymbol{\alpha}:(\boldsymbol{N} * b), \quad q=\frac{1}{\rho_{0} c_{\sigma}} \operatorname{div} \boldsymbol{q}, \\
& m_{\sigma}=\frac{1}{\rho_{0} c_{\sigma}}\left[\gamma_{0}\left(\sigma: \boldsymbol{F}_{\sigma}-\Pi \cdot b\right)-\gamma_{3} T\left(\frac{\partial \Pi\left(Y_{K}^{T \sigma}\right)}{\partial T} \cdot b\right)\right] .
\end{aligned}
$$

The set of algebraic Eqs (A.1) has a unique solution in the form

$$
\left\{\begin{array}{l}
H \Lambda^{(p)}=F_{\sigma}: \boldsymbol{M} \dot{\boldsymbol{\varepsilon}}-\frac{1}{p}\left(F_{T}-\gamma_{5} \boldsymbol{\alpha}: \boldsymbol{M F}_{\boldsymbol{\sigma}}\right)\left(\bar{\gamma}_{6} \xi \boldsymbol{\alpha}: \boldsymbol{M} \dot{\boldsymbol{\varepsilon}}+q\right), \\
H \dot{T}^{(p)}=\frac{1}{p}\left(m_{\varepsilon}+\bar{\gamma}_{6} \xi \boldsymbol{\alpha}: \boldsymbol{M \boldsymbol { F } _ { \sigma }}\right)\left(\boldsymbol{F}_{\boldsymbol{\sigma}}: \boldsymbol{M} \dot{\boldsymbol{\varepsilon}}\right)-\frac{h^{*}}{p}\left(\bar{\gamma}_{6} \xi \boldsymbol{\alpha}: \boldsymbol{M} \dot{\boldsymbol{\varepsilon}}+q\right),
\end{array}\right.
$$

where

$$
h^{*}=h+g_{p}: M F_{\sigma},
$$

provided that the condition $H \neq 0$. Now it will be assumed that unloading takes place for the prescribed values of $\dot{\varepsilon}$ and $\operatorname{div} \boldsymbol{q}$ and $\left(\dot{\boldsymbol{\varepsilon}}^{p}=0\right)$. Let us denote by $\dot{T}^{(e)}$ and $\dot{\boldsymbol{\sigma}}^{(e)}$ the relevant values for that process.

$$
\left\{\begin{array}{l}
\dot{T}^{(e)}=\frac{1}{p}\left(\bar{\gamma}_{6} \xi \alpha: M \dot{\varepsilon}+q\right) \\
\dot{\boldsymbol{\sigma}}^{(e)}=\boldsymbol{M} \dot{\varepsilon}+\frac{\gamma_{5}}{p}\left(\bar{\gamma}_{6} \xi \alpha: M \dot{\varepsilon}+q\right) \boldsymbol{M \alpha}
\end{array}\right.
$$

Making use of (A.3), we can establish the following relation between $L^{(e)}=\boldsymbol{F}_{\boldsymbol{\sigma}}: \dot{\boldsymbol{\sigma}}^{(e)}+F_{T} \dot{T}$ and $\Lambda^{(p)}$ as

$$
H \Lambda^{(p)}=L^{(e)}
$$


In agreement with the unloading criterion assumed, which is that of the expression of $L^{(e)}$ being negative, we have the relation $\left(\operatorname{sign} \Lambda^{(p)}=\operatorname{sign} L^{(e)}\right.$ ), which ensures the existence and uniqueness of $\dot{\sigma}$. Then, from Eq.(A.5) we find that

$$
H>0 .
$$

2. The procedure of deriving the condition $h_{1}>0$ (problem $\boldsymbol{b}_{1}$ ) is analogous to that for the first problem, except that in the present case it is more convenient to use Eqs (2.1). We are interested only in those points of the body where $F=0$. The quantities $\Lambda^{(p)}$ and $\dot{T}^{(p)}$ and also $\dot{T}^{(e)}$ are now the functions of $\dot{\sigma}$ . Let us assume that for given values of $\dot{\sigma}$ and $\operatorname{div} \boldsymbol{q}$ the loading process occurs $\left(\dot{\boldsymbol{\varepsilon}}^{p} \neq 0\right)$. From Eqs ( $\left.\dot{\boldsymbol{\varepsilon}}=\dot{\boldsymbol{\varepsilon}}^{e}+\dot{\boldsymbol{\varepsilon}}^{p}\right),(2.1)_{1},(2.10)_{1}$ and $(2.10)_{2}$ we find

$$
\left\{\begin{array}{l}
F_{T} \dot{T}^{(p)}-h_{1} \Lambda^{(p)}=-\boldsymbol{F}: \dot{\boldsymbol{\sigma}}, \\
\dot{T}^{(p)}-m_{\sigma} \Lambda^{(p)}=-\bar{\gamma}_{7} \xi \boldsymbol{\alpha}: \dot{\boldsymbol{\sigma}}-q .
\end{array}\right.
$$

This alternative set of algebraic Eqs (A.7) has a unique solution in the form

$$
\left\{\begin{array}{l}
h_{1} \Lambda^{(p)}=\boldsymbol{F}_{\sigma}: \dot{\sigma}-\bar{\gamma}_{7} F_{T} \xi \alpha: \dot{\sigma}-q F_{T}, \\
h_{1} \dot{T}^{(p)}=m_{\sigma} \boldsymbol{F}_{\sigma}: \dot{\sigma}-\bar{\gamma}_{7} h \xi \alpha: \dot{\sigma}-h q,
\end{array}\right.
$$

provided that $h_{l}=h-m_{\sigma} F_{T} \neq 0$.

Let us assume, for prescribed $\dot{\sigma}$ and $\operatorname{div} \boldsymbol{q}$, that the process of unloading takes place $\left(\dot{\boldsymbol{\varepsilon}}^{p}=0\right)$. Then from $(2.1)_{1}$, we obtain immediately

$$
\left\{\begin{array}{l}
\dot{T}^{(e)}=-\bar{\gamma}_{7} \xi \alpha: \dot{\sigma}-q, \\
\dot{\boldsymbol{\varepsilon}}^{(e)}=\boldsymbol{L} \dot{\sigma}-\gamma_{4}\left(\bar{\gamma}_{7} \xi \alpha: \dot{\sigma}-q\right) \alpha \\
\dot{\boldsymbol{\varepsilon}}=\dot{\boldsymbol{\varepsilon}}^{(e)}
\end{array}\right.
$$

By evaluating $L^{(e)}=\boldsymbol{F}_{\boldsymbol{\sigma}}: \dot{\sigma}+F_{T} \dot{T}^{(e)}$ it is easily observed that

$$
h_{1} \Lambda^{(p)}=L^{(e)}
$$

Hence, the condition sought for is

$$
h_{1}>0 \text {. }
$$




\subsection{Apendix B}

The derivation of the sufficient local uniqueness condition for the solution of an incremental boundary-value problem has been modelled according to the described in (Śloderbach, [1], [2], [30]; Raniecki, [4], [5]; Raniecki and Bruhns, [6]).

Let assume that there are two sets of functions $\{\dot{\boldsymbol{\sigma}}, \dot{\boldsymbol{\varepsilon}}, \dot{\boldsymbol{u}}, \dot{T}\}$ and $\left\{\dot{\boldsymbol{\sigma}}^{*}, \dot{\boldsymbol{\varepsilon}}^{*}, \dot{\boldsymbol{u}}^{*}, \dot{T}\right\}$, which are solutions of the incremental (rate type) boundary-value problem. Our task is to find the condition for $\left(\dot{\sigma}=\dot{\sigma}^{*}\right)$ and functions $\left(\dot{\boldsymbol{\varepsilon}}=\dot{\boldsymbol{\varepsilon}}^{*}\right)$ and $\left(\dot{T}=\dot{T}^{*}\right)$ are obtained in a unique manner from Eqs $(2.10)_{1}$ and $(2.10)_{2}$. The satisfaction of this condition implies that $h_{1}>0$.

By applying the Gauss-Ostrogradsky theorem we have

$$
\int_{D}\left(\dot{\sigma}-\dot{\sigma}^{*}\right):\left(\dot{\varepsilon}-\dot{\varepsilon}^{*}\right) d V=0
$$

Acciording to Eq.(2.10), $\dot{\varepsilon}$ and $\dot{\varepsilon}^{*}$ are functions of $\dot{\sigma}$ and $\dot{\sigma}^{*}$, respectively. Let us denote the integrand Eq.(B.1) as follows

$$
I\left(\dot{\sigma}, \dot{\sigma}^{*}, j, j^{*}\right)=\Delta \dot{\sigma}: \Delta \dot{\varepsilon}
$$

where $\Delta \dot{\sigma}=\left(\dot{\sigma}-\dot{\sigma}^{*}\right)$ and $\Delta \dot{\varepsilon}=\left(\dot{\varepsilon}-\dot{\varepsilon}^{*}\right)$ and $j=j(\dot{\sigma})$ and $j=\left(\dot{\sigma}^{*}\right)$ are determined by Eq. $(2.11)_{3}$.

Bering this in mind we must find a condition for $I$ to be a positive definite function (that is $I>0$ for $\left(\dot{\sigma} \neq \dot{\sigma}^{*}\right)$ and $I=0$ for $\left(\dot{\sigma}=\dot{\sigma}^{*}\right)$ ). Let us observe that Eq. $(2.10)_{1}$ can be written in the form

$$
\dot{\boldsymbol{\varepsilon}}=\boldsymbol{L}_{l} \dot{\sigma}+\frac{j}{h_{l}} \boldsymbol{g}\left(\overline{\boldsymbol{F}}_{\sigma}: \dot{\sigma}+Z_{l}\right)+\boldsymbol{d}_{1}
$$

where

$$
\left\{\begin{array}{l}
\boldsymbol{g}=\left(\boldsymbol{F}_{1, \boldsymbol{\sigma}}+\bar{\gamma}_{9} \boldsymbol{Z} * b+\gamma_{4} m_{\sigma} \boldsymbol{\alpha}\right), \quad \overline{\boldsymbol{F}}_{\sigma}=\left(\boldsymbol{F}_{\sigma}-\bar{\gamma}_{7} \xi F_{T} \boldsymbol{\alpha}\right), \\
d_{l}=-\gamma_{4} q \boldsymbol{\alpha}, Z_{1}=-q F_{T}, \quad \boldsymbol{L}_{l}=\boldsymbol{L}^{\boldsymbol{a}}, \quad \boldsymbol{M}_{\boldsymbol{1}}=\boldsymbol{M}^{\boldsymbol{a}}
\end{array}\right.
$$

a) Let $j=j^{*}=1$. On substituting (B.3) into (B.2) $)_{1}$ we find

$$
I_{1}=I\left(\dot{\sigma}, \dot{\sigma}^{*}, 1,1\right)=\Delta \dot{\boldsymbol{\sigma}}: \boldsymbol{L}_{1} \Delta \dot{\boldsymbol{\sigma}}+\frac{1}{h_{l}}\left[(g: \Delta \dot{\boldsymbol{\sigma}})\left(\overline{\boldsymbol{F}}_{\sigma}: \Delta \dot{\boldsymbol{\sigma}}\right)\right]
$$

Let us resolve the vector $\Delta \dot{\sigma}$ into components, the directions of which are those of $\boldsymbol{M}_{1} \boldsymbol{g}$ and $\boldsymbol{M}_{1} \overline{\boldsymbol{F}}_{\boldsymbol{\sigma}}$ and the direction $t$ normal to the other two in a 9-dimensional space with a metric $\boldsymbol{L}_{l}$. By evaluating the product $\left(\Delta \dot{\boldsymbol{\sigma}}: \boldsymbol{L}_{1} \Delta \dot{\boldsymbol{\sigma}}\right)$ we find that the expression for $I_{1}$ (B.5) takes the form 


$$
I_{l}=\left\{\begin{array}{l}
\boldsymbol{t}: \boldsymbol{L}_{l} \boldsymbol{t}+\frac{1}{h_{l}\left(1-m_{g f}^{2}\right)}\left[A\left(\overline{\boldsymbol{F}}_{\boldsymbol{\sigma}}: \Delta \dot{\boldsymbol{\sigma}}\right)^{2}+2 B\left(\overline{\boldsymbol{F}}_{\boldsymbol{\sigma}}: \Delta \dot{\boldsymbol{\sigma}}\right)(\boldsymbol{g}: \Delta \dot{\boldsymbol{\sigma}})+C(\boldsymbol{g}: \Delta \dot{\boldsymbol{\sigma}})^{2}\right], \\
\text { if } m_{g f}^{2}<1, \\
\boldsymbol{t}: \boldsymbol{L}_{l} \boldsymbol{t}+\frac{A_{0}}{M_{f}^{2} h_{l}}\left(\overline{\boldsymbol{F}}_{\boldsymbol{\sigma}}: \Delta \dot{\boldsymbol{\sigma}}\right)^{2}, \quad \text { if } \quad m_{g f}^{2}=1,
\end{array}\right.
$$

where

$$
\left\{\begin{array}{l}
\boldsymbol{t}=\Delta \dot{\boldsymbol{\sigma}}-\frac{1}{\left(1-m_{g f}^{2}\right)}\left[\frac{1}{M_{f}^{2}}\left(\overline{\boldsymbol{F}}_{\boldsymbol{\sigma}}: \Delta \dot{\boldsymbol{\sigma}}\right)-\frac{m_{g f}}{M_{f} M_{g}}(\boldsymbol{g}: \Delta \dot{\boldsymbol{\sigma}})\right] \boldsymbol{M}_{\boldsymbol{l}} \overline{\boldsymbol{F}}_{\boldsymbol{\sigma}}+ \\
-\frac{1}{\left(1-m_{g f}^{2}\right)}\left[\frac{1}{M_{g}^{2}}(\boldsymbol{g}: \Delta \dot{\boldsymbol{\sigma}})-\frac{m_{g f}}{M_{f} M_{g}}\left(\overline{\boldsymbol{F}}_{\boldsymbol{\sigma}}: \Delta \dot{\boldsymbol{\sigma}}\right)\right] \boldsymbol{M}_{\boldsymbol{1}} \boldsymbol{g}, \text { if } m_{g f}^{2}<1, \\
\boldsymbol{t}=\Delta \dot{\boldsymbol{\sigma}}-\frac{\left(\overline{\boldsymbol{F}}_{\boldsymbol{\sigma}}: \Delta \dot{\boldsymbol{\sigma}}\right)}{M_{f}^{2}} \boldsymbol{M}_{\boldsymbol{1}} \overline{\boldsymbol{F}}_{\boldsymbol{\sigma}}, \quad \text { if } m_{g f}^{2}<1, \\
\text { and } M_{f}^{2}=\overline{\boldsymbol{F}}_{\sigma}: \boldsymbol{M}_{\boldsymbol{1}} \overline{\boldsymbol{F}}_{\boldsymbol{\sigma}}, \quad M_{g}^{2}=\boldsymbol{g}: \boldsymbol{M}_{\boldsymbol{1}} \boldsymbol{g}, \quad m_{g f}^{2}=\frac{\boldsymbol{g}: \boldsymbol{M}_{\boldsymbol{1}} \overline{\boldsymbol{F}}_{\boldsymbol{\sigma}}}{M_{f} M_{g}} \\
A=\frac{h_{l}}{M_{f}^{2}}, B=\frac{1}{2}\left(1-m_{g f}^{2}\right)-\frac{m_{g f}}{M_{f} M_{g}} h_{l} C=\frac{h_{1}}{M_{g}^{2}}, \\
\text { as well } A_{0}=h_{l}+m_{g f} M_{f} M_{g} .
\end{array}\right.
$$

Since $h_{1}>1$, therefore $I_{l}$ (B.6) is a symmetric quadratic form, which is definite positive, if

$$
A C-B^{2}>0 \text {. }
$$

On substituting (B.7) into (B.8), we find, in a similar manner, that

$$
a_{1} h_{l}^{2}+b_{1} h_{1}+c_{1}>0
$$

where $\quad a_{1}=1, \quad b_{1}=m_{g f} M_{f} M_{g}, \quad c_{1}=-\left(1-m_{g f}^{2}\right) \frac{M_{f}^{2} M_{g}^{2}}{4}$.

On solving the above inequality (B.9), we find the condition

$$
h_{l}>\frac{1}{2} M_{f} M_{g}\left(1-m_{g f}\right) \text {. }
$$


On substituting Eq.(B.7) in the the above expression (B.10) we obtain easily condition (4.1). Let us add that in the case of $\left(\bar{\gamma}_{7}=\gamma_{4}=\bar{\gamma}_{9}=\gamma_{12}=0\right)$ condition (B.10) or expression (4.1) take the form

$$
h_{1}>0
$$

b) Let $j=1, j^{*}=0$. Then the expression for $I_{2}$ has the form

$$
I_{2}=I\left(\dot{\boldsymbol{\sigma}}, \dot{\sigma}^{*}, 1,0\right)=\Delta \dot{\boldsymbol{\sigma}}: \boldsymbol{L}_{1} \Delta \dot{\boldsymbol{\sigma}}+\frac{1}{h_{1}}\left[(\boldsymbol{g}: \Delta \dot{\boldsymbol{\sigma}})\left(\overline{\boldsymbol{F}}_{\boldsymbol{\sigma}}: \dot{\boldsymbol{\sigma}}+Z_{1}\right)\right] .
$$

The study of expression (B.12) for positive definiteness will be as follows. Let us resolve $g$ in the directions $\overline{\boldsymbol{F}}_{\sigma}$ and $\boldsymbol{\beta}$ in the following manner

$$
\boldsymbol{g}=c \overline{\boldsymbol{F}}_{\sigma}+\boldsymbol{\beta}
$$

where $c$ is a parameter to be used for optimizing the uniqueness condition in the case of simultaneous loading and unloading $\left(j=1\right.$ and $j^{*}=0$ ). Then from Eqs (B.4) and (B.13) we find

$$
\boldsymbol{\beta}=\overline{\boldsymbol{F}}_{\boldsymbol{\sigma}}(1-c)+\left(\gamma_{4} m_{\sigma}+c \bar{\gamma}_{7} \xi F_{T}\right) \boldsymbol{\alpha}+\boldsymbol{Z} *\left(\gamma_{12} F_{1, \Pi}+\gamma_{9} b\right) .
$$

Then expression (B.12) must be expressed as a quadratic form. Let estimate therefore $I^{2}$ as follows

$$
I_{2} \geq I_{2}^{\prime}=\Delta \dot{\boldsymbol{\sigma}}: \boldsymbol{L}_{1} \Delta \dot{\boldsymbol{\sigma}}+\frac{1}{h_{1}}\left[c \bar{A}_{\sigma}^{2}+\bar{A}_{\sigma}(\boldsymbol{\beta}: \Delta \dot{\boldsymbol{\sigma}})\right]
$$

where

$$
\left\{\begin{array}{l}
I_{2}=I_{2}^{\prime}-\frac{c}{h_{1}} \bar{A}_{\sigma} \bar{A}_{\sigma}^{*}, \\
\bar{A}_{\sigma}=\overline{\boldsymbol{F}}_{\sigma}: \dot{\sigma}+Z_{1} \geq 0, \quad \bar{A}_{\sigma}^{*}=\overline{\boldsymbol{F}}_{\sigma}: \dot{\sigma}^{*}+Z_{1}<0 .
\end{array}\right.
$$

Let us resolve, as before, the vector $\Delta \dot{\sigma}$ in the directions $\boldsymbol{M}_{l} \boldsymbol{\beta}$ and the direction $\boldsymbol{t}_{l}$ normal to $\boldsymbol{M}_{l} \boldsymbol{\beta}$ in a 9-dimensional space with a metric $\boldsymbol{L}_{l}$. On substituting the result thus obtained into (B.15) we find that

$$
I_{2}^{\prime}=\boldsymbol{t}: \boldsymbol{L}_{1} \boldsymbol{t}+\frac{1}{h_{1}}\left[\bar{A}_{1}(\boldsymbol{\beta}: \Delta \dot{\boldsymbol{\sigma}})^{2}+2 \bar{B}_{1} \bar{A}_{\sigma}(\boldsymbol{\beta}: \Delta \dot{\boldsymbol{\sigma}})+\bar{C}_{1} \bar{A}_{\sigma}^{2}\right]
$$

where

$$
\left\{\begin{array}{l}
\boldsymbol{t}_{l}=\Delta \dot{\boldsymbol{\sigma}}-\frac{\boldsymbol{\beta}: \Delta \dot{\boldsymbol{\sigma}}}{M_{\beta}^{2}} \boldsymbol{M}_{l} \boldsymbol{\beta}, \quad M_{\beta}^{2}=\boldsymbol{\beta}: \boldsymbol{M}_{l} \boldsymbol{\beta}, \\
\bar{A}_{l}=\frac{h_{l}}{M_{\beta}^{2}}, \quad \bar{B}_{l}=\frac{1}{2}, \quad \bar{C}_{l}=c .
\end{array}\right.
$$

Expression (B.17) is a positive quadratic form, if 


$$
\bar{A}_{1} \bar{C}_{1}-\bar{B}_{1}^{2}>0
$$

On substituting expression (B.18) into (B.19) we find

$$
h_{l}=\frac{M_{\beta}^{2}}{4 c} .
$$

The form of expression (B.20) shows that we have a one-parameter family of uniqueness conditions, the parameter being $c$. We want to determine the condition of minimum with respect to $c$, to be able to estimate the bifurcation states as closely as possible.

On substituting Eq.(B.13) in Eq.(B.20), we obtain

$$
h_{l}>\frac{1}{4 c}\left[\left(\boldsymbol{g}-c \overline{\boldsymbol{F}}_{\boldsymbol{\sigma}}\right): \boldsymbol{M}_{l}\left(\boldsymbol{g}-c \overline{\boldsymbol{F}}_{\boldsymbol{\sigma}}\right)\right]=\frac{1}{4 c}\left[M_{g}^{2}-2 c M_{g f}+c^{2} M_{f}^{2}\right],
$$

where

$$
M_{g f}=\boldsymbol{g}: \boldsymbol{M}_{1} \overline{\boldsymbol{F}}_{\sigma}=\overline{\boldsymbol{F}}_{\sigma}: \boldsymbol{M}_{1} \boldsymbol{g}
$$

The right-hand side of Eq.(B.21) must, therefore, become minimum in the scalar parameter $c$. Let

$$
F(c)=\frac{1}{4}\left(\frac{M_{g}^{2}}{c}-2 M_{g f}+c M_{f}^{2}\right),
$$

then

$$
\frac{\partial F(c)}{\partial c}=0
$$

From condition (B.23) we find that

$$
c_{0}=\frac{M_{g}}{M_{f}}, \quad F\left(c_{0}\right)=F_{0}, \quad \text { and } \quad c>0 .
$$

On substituting Eq.(B.24) $)_{1}$ into inequality (B.21) we obtain, after some manipulation, that

$$
h_{1}>\frac{1}{2}\left(M_{g} M_{f}-M_{g f}\right) \text {. }
$$

By introducing the notations (B.7) we obtain easily expression (4.1) sought-for.

It can be shown that the right-hand member of Eq.(B.17) is zero, if

$$
\bar{A}_{l}(\beta: \Delta \dot{\sigma})=-\bar{B}_{l} \bar{A}_{\sigma} \quad \text { and } \quad \bar{B}_{l}(\beta: \Delta \dot{\sigma})=-\bar{C} A_{\sigma} .
$$

Hence, $I_{2}=I_{2}^{\prime}$ if $\bar{A}_{\sigma}=0$. Then it follows from Eqs (B.16) - (B.26) that $I_{2}>0$.

c) Let $j=0, j^{*}=1$. The procedure of demonstrating that

$$
I_{3}=I\left(\dot{\sigma}, \dot{\sigma}^{*}, 0,1\right)>0
$$

is analogous to that in the case (b). We have, therefore, 


$$
I_{3}^{\prime}=I_{3}+\frac{c}{h_{1}} \bar{A}_{\sigma} \bar{A}_{\sigma}^{*}
$$

where

$$
\bar{A}_{\sigma}=\overline{\boldsymbol{F}}_{\sigma}: \dot{\sigma}+Z_{l}<0 \text { and } \bar{A}_{\sigma}^{*}=\overline{\boldsymbol{F}}_{\sigma}: \dot{\sigma}^{*}+Z_{l} \geq 0
$$

Hence,

$$
I_{3}^{\prime} \geq I_{3}=\boldsymbol{t}: \boldsymbol{L}_{l} \boldsymbol{t}+\frac{1}{h_{1}}\left[\bar{A}_{l}(\boldsymbol{\beta}: \Delta \dot{\boldsymbol{\sigma}})^{2}-2 \bar{B}_{1} \bar{A}_{\sigma}(\boldsymbol{\beta}: \Delta \dot{\boldsymbol{\sigma}})+\bar{C}_{l}\left(\bar{A}_{\sigma}^{*}\right)^{2}\right]
$$

where $\left(\boldsymbol{t}_{1}, \bar{A}_{1}, \bar{B}_{1}\right.$ and $\left.\bar{C}_{1}\right)$ are defined by Eq.(B.18).

Similarly to the case (b) it follows that $I_{3}>0$.

d) Let $j=j^{*}=0$. Then the integrand

$$
I_{4}=I\left(\dot{\boldsymbol{\sigma}}, \dot{\boldsymbol{\sigma}}^{*}, 0,0\right)=\Delta \dot{\boldsymbol{\sigma}}: \boldsymbol{L}_{1} \Delta \dot{\boldsymbol{\sigma}}
$$

is positive definite because $L_{l}=L^{a}$ is positive definite. Then from (B.1) it follows directly that $\dot{\sigma}=\dot{\sigma}^{*}$.

\subsection{Apendix C}

It will now be shown that $J^{\prime}$ is positively definite if expression (4.1) is satisfied. It will be shown that the sufficient local uniqueness condition for the integrand $J^{\prime}$ is the same as in the case of an generalized thermo-elastic-plastic body $J$ (4.4). It constitutes also a criterion enabling us to confirm the reason for introducting the expression $J^{\prime}$. Let us denote

$$
\left(\boldsymbol{g}^{*}+x^{2} \overline{\boldsymbol{F}}_{\sigma}^{*}\right)=\boldsymbol{M}_{\boldsymbol{l}} \boldsymbol{W} .
$$

From Eq.(4.6) (Part I) and (C.1) after transformations it follows that

$$
\left\{\begin{array}{l}
\boldsymbol{M}_{\boldsymbol{I}} \boldsymbol{W}=\varphi(\boldsymbol{M} \boldsymbol{\alpha})-\boldsymbol{N}_{\boldsymbol{Z}}+\boldsymbol{B}\left(1+x^{2}\right), \\
\boldsymbol{W}=\varphi \boldsymbol{L}_{l}(\boldsymbol{M} \boldsymbol{\alpha})-\boldsymbol{L}_{\boldsymbol{l}} \boldsymbol{N}_{\boldsymbol{Z}}+\boldsymbol{L}_{\boldsymbol{l}} \boldsymbol{B}\left(1+x^{2}\right) .
\end{array}\right.
$$

On substituting Eq.(C.1) into (4.7), we obtain

$$
J^{\prime}=\Delta \dot{\boldsymbol{\varepsilon}}: \boldsymbol{M}_{1} \Delta \dot{\boldsymbol{\varepsilon}}-\frac{1}{4 x^{2} H_{l}}\left[\left(\boldsymbol{M}_{l} \boldsymbol{W}\right): \Delta \dot{\boldsymbol{\varepsilon}}\right]^{2} .
$$

On resolving $\Delta \dot{\varepsilon}$ onto the directions $\boldsymbol{W}$ and $\boldsymbol{t}^{*}$ normal to $\boldsymbol{W}$ in a 9-dimensional space with a metric $\boldsymbol{M}_{1}$, we obtain the expression

$$
\boldsymbol{t}^{*}=\Delta \dot{\varepsilon}-\frac{\Delta \dot{\varepsilon}:\left(M_{1} \boldsymbol{W}\right)}{M_{w}^{2}} \boldsymbol{W}
$$


where $M_{w}^{2}=\boldsymbol{W}: \boldsymbol{M}_{1} \boldsymbol{W}$.

Then $J^{\prime}$ (C.3) will have the form

$$
J^{\prime}=\boldsymbol{t}^{*}: \boldsymbol{M}_{1} \boldsymbol{t} *+\frac{1}{4 x^{2} H_{1}}\left(4 x^{2} H_{1}-M_{w}^{2}\right)\left(\Delta \dot{\varepsilon}: \boldsymbol{M}_{l} \boldsymbol{W}\right)
$$

From (C.5) it follows that $J^{\prime}$ is positive definite, if

$$
H_{1}>\frac{1}{4 x^{2}}\left(\boldsymbol{W}: \boldsymbol{M}_{1} \boldsymbol{W}\right)
$$

Condition (C.6) must now be optimized by finding the minimum in the parameter $x^{2}$. Condition (C.6) yields a one-parameter family of sufficient uniqueness conditions for a comparison body.

Let: $\left(y=x^{2}\right)$, hence $y>0$.

Then

$$
P(y)=\frac{1}{y}\left(\boldsymbol{W}: \boldsymbol{M}_{\boldsymbol{1}} \boldsymbol{W}\right) .
$$

Calculating the derivative of Eq.(C.7) and likening it to zero, we obtain

$$
\left\{\begin{array}{l}
\frac{\partial P(y)}{\partial y}=-\frac{1}{y^{2}}\left(\boldsymbol{W}: \boldsymbol{M}_{1} \boldsymbol{W}\right)+\frac{2}{y}\left(\boldsymbol{W}: \boldsymbol{M}_{1} \boldsymbol{L}_{\boldsymbol{l}} \boldsymbol{B}\right)=0 \\
\text { then } \\
2 y(\boldsymbol{W}: \boldsymbol{B})=\left(\boldsymbol{W}: \boldsymbol{M}_{1} \boldsymbol{W}\right) .
\end{array}\right.
$$

The expression (C.8 $)_{2}$ results from the condition of the function $P(y)$ taking an extremum value in the scalar parameter $y$. Expression (C.8 $)_{2}$ must be transformed to obtain the desired result, the expressions for $\boldsymbol{W}$ (cf. Eq.(C.2) 1 ) being taken into account. Let us rewrite expression (C.2 $)_{2}$ in the form

$$
\boldsymbol{W}=\boldsymbol{W}_{1}+x^{2} \boldsymbol{L}_{\mathbf{l}} \boldsymbol{B}
$$

where

$$
W_{l}=\varphi L_{1}(M \alpha)-L_{1} N_{z}+L_{1} B
$$

on substituting Eq.(C.9) into (C.8), we find, after rearrangement

$$
y=x_{0}^{2}=\sqrt{\frac{\boldsymbol{g}^{*}: \boldsymbol{L}_{\mathbf{1}} \boldsymbol{g}^{*}}{\overline{\boldsymbol{F}}_{\sigma}^{*}: \boldsymbol{L}_{\mathbf{1}} \overline{\boldsymbol{F}}_{\sigma}^{*}}}
$$

where $\boldsymbol{g}^{*}$ and $\overline{\boldsymbol{F}}_{\sigma}^{*}$ are defined by relations (4.6), and (4.6) $)_{2}$, respectively.

It can be easily shown that by substituting Eq.(C.10) into (C.6) and taking into consideration (4.6) and (C.9) we shall obtain, after rearrangement 


$$
H_{1}>\frac{1}{2}\left[\sqrt{\left(\boldsymbol{W}_{1}: \boldsymbol{M}_{1} \boldsymbol{W}_{1}\right)\left(\boldsymbol{B}: \boldsymbol{L}_{1} \boldsymbol{B}\right)}+\boldsymbol{W}_{\mathbf{l}}: \boldsymbol{B}\right] .
$$

It can also be shown that

$$
\boldsymbol{W}_{1}=\boldsymbol{g} \quad \text { and } \quad \boldsymbol{B}=\boldsymbol{M}_{1} \overline{\boldsymbol{F}}_{\sigma}
$$

Then, from Eqs (2.5), (2.6), (4.2) and (C.12) it follows that

$$
H=h_{1}+\boldsymbol{g}: \boldsymbol{M}_{\mathbf{1}} \overline{\boldsymbol{F}}_{\sigma} .
$$

On substituting Eq.(C.12) and (C.13) into (C.11) we find easily, after rearrangement, condition (4.1), our proof. thus being accomplished.

\subsection{Apendix D}

It will be shown by analogy (Appendix C) that $I^{\prime}$ is positive definite if inequality (4.1) is satisfied. This will be show that the sufficient local uniqueness condition for the integrand $J^{\prime}$ is the same as in the case of an generalized thermo-elastic-plastic body $I$ (4.16). It constitutes also a criterion enabling us to confirm the reason for introducting the expression $I^{\prime}$.

Let us denote on the basis of (4.15) that

$$
\left(\boldsymbol{g}-y^{2} \overline{\boldsymbol{F}}_{\sigma}\right) \equiv \boldsymbol{L}_{\boldsymbol{I}} \boldsymbol{W}_{\boldsymbol{l}}
$$

From expression (C.1) we will that after sliding tensor $\boldsymbol{M}_{l}$ receive that

$$
\boldsymbol{M}_{l}\left(\boldsymbol{g}-\boldsymbol{y}^{2} \overline{\boldsymbol{F}}_{\sigma}\right) \equiv \boldsymbol{W}_{1}
$$

where $\boldsymbol{M}_{l}=\left.\left(\boldsymbol{L}_{l}^{-1}\right)\right|_{\sigma=\sigma(T, \varepsilon, K)}$ but expressions on $\boldsymbol{g}$ and $\overline{\boldsymbol{F}}_{\sigma}$ follow from Eq.(4.2).

On substituting Eq.(D.1) into (4.15), we obtain

$$
I^{\prime}=\Delta \dot{\sigma}: \boldsymbol{L}_{l} \Delta \dot{\boldsymbol{\sigma}}-\frac{1}{4 y^{2} h_{1}}\left(\Delta \dot{\boldsymbol{\sigma}}: \boldsymbol{L}_{\boldsymbol{l}} \boldsymbol{W}_{\boldsymbol{l}}\right)^{2}
$$

On resolving $\Delta \dot{\sigma}$ onto the directions $\boldsymbol{W}_{l}$ and $\boldsymbol{t}^{*}$ normal to $\boldsymbol{W}_{l}$ in a 9-dimensional space with a metric $\boldsymbol{L}_{l}$, we obtain the expression

$$
\boldsymbol{t}_{1}=\Delta \dot{\boldsymbol{\sigma}}-\frac{\left(\boldsymbol{L}_{\boldsymbol{l}} \boldsymbol{W}_{\boldsymbol{1}}\right): \Delta \dot{\boldsymbol{\sigma}}}{L_{w}^{2}}
$$

where

$$
L_{w}^{2}=W_{1}: L_{1} W_{1} .
$$

Then 


$$
I^{\prime}=\boldsymbol{t}_{l}: \boldsymbol{L}_{l} \boldsymbol{t}_{1}+\frac{1}{4 y^{2} h_{l}}\left(4 y^{2} h_{l}-L_{w}^{2}\right)\left(\Delta \dot{\boldsymbol{\sigma}}: \boldsymbol{L}_{\mathbf{l}} \boldsymbol{W}_{\boldsymbol{l}}\right)
$$

From (D.5) it follows that $I$ ' is positive definite, if

$$
h_{1}>\frac{1}{4 y^{2}}\left(\boldsymbol{W}_{1}: \boldsymbol{L}_{1} \boldsymbol{W}_{1}\right) \text {. }
$$

Condition (C.6) yields a one-parameter family on account of the $y^{2}$ parameter of sufficient uniqueness conditions for a comparative body. Condition (D.6) must now be optimized by finding the minimum in the parameter $y^{2}$.

Let $\left(r \equiv y^{2}\right)$, hence $r>0$.

Then

$$
P(r)=\frac{1}{4 r}\left(W_{1}: L_{1} W_{1}\right)
$$

Substituting $\left(r \equiv y^{2}\right)$ in Eqs (D.2) and (D.7) and likening the calculated derivative to zero, we receive

therefore

$$
\frac{\partial P(r)}{\partial r}=-\frac{1}{r^{2}}\left(\boldsymbol{W}_{\boldsymbol{1}}: \boldsymbol{L}_{\boldsymbol{1}} \boldsymbol{W}_{\boldsymbol{1}}\right)-\frac{2}{r}\left(\boldsymbol{W}_{\boldsymbol{1}}: \boldsymbol{L}_{1} \boldsymbol{M}_{1} \overline{\boldsymbol{F}}_{\boldsymbol{\sigma}}\right)=0,
$$

$$
-2 r\left(W_{1}: \bar{F}_{\sigma}\right)=\left(W_{1}: L_{1} W_{1}\right)
$$

Substituting $\boldsymbol{g}$ and $\overline{\boldsymbol{F}}_{\sigma}$ determined by expressions (4.2) and ( $\left.r \equiv y^{2}\right)$ into Eq.(D.9), after conversions, yields

$$
y_{0}^{2}=\sqrt{\frac{g: M_{1} g}{\overline{\boldsymbol{F}}_{\sigma}: M_{1} \overline{\boldsymbol{F}}_{\sigma}}} .
$$

This is a similar expression received earlier in papers (Śloderbach, [1], [2], [30]; Raniecki, [4], [5]; Raniecki and Bruhns, [6]; Śloderbach and Pajak, [28]). It can be shown that, by substituting Eqs (D.2), (D.10) into (D.6) and taking into consideration (4.2) we shall obtain, after rearrangement, that

$$
h_{1}>\frac{1}{2}\left[\sqrt{\left(\boldsymbol{g}: \boldsymbol{M}^{(a)} \boldsymbol{g}\right)\left(\overline{\boldsymbol{F}}_{\sigma}: \boldsymbol{M}^{(a)} \overline{\boldsymbol{F}}_{\boldsymbol{\sigma}}\right)}-\boldsymbol{g}: \boldsymbol{M}^{(a)} \overline{\boldsymbol{F}}_{\boldsymbol{\sigma}}\right]=h_{l}^{c r} .
$$

Let us note that this expression is analogical to the expression obtained for the case of a generalized coupled elastic-thermoplastic body, see relationship (4.1). This mathematical fact proves that introduction of such an integrand $I$ ' for the reference body dependent on statically admissible stress velocity fields is right.

Finally, we can state once again that condition (D.11) is also the necessary and sufficient condition if the expressions $J$, see (4.4) and $J$ ', see (4.7), and the expressions $I$, see. (4.12), $I$, see (4.15), may be positively defined.

\section{Conclusions}

1. In the paper, the necessary and sufficient conditions of uniqueness of solution of the formulated incremental boundary problem of coupled generalized thermoplasticity for small gradients of 
displacements (small strains), were derived. Global sufficient conditions and also local sufficient conditions (safer for small strains) were derived. Conditions of uniqueness for the generalized thermoplastic body, see (Śloderbach, [1], [2], [3], [7], [30]) and for suitable comparison bodies were determined. The derived conditions of uniqueness (global and local) are suitable necessary and sufficient criteria excluding bifurcation of equilibrium states of coupled generalized thermoplasticity, also in isothermal loading processes. It was also shown that the local conditions of uniqueness for the generalized thermoplastic bodies and the comparison bodies have the same form. Thus, introduction of such comparison bodies seems to be proper. But the global conditions of uniqueness and the global criteria of bifurcation have different forms.

2. There are papers concerning problems of solution of uniqueness and bifurcation of equilibrium states (see Raniecki and Mróz, [4], [5]; Raniecki, [6], [33]; Raniecki and Sawczuk, [36]; Raniecki and Bruhns, [34]; Śloderbach, [1], [3]). In this paper, however, a new global and local criterion was formulated for the derived comparison body dependent on statically permissible velocity fields of stress. Thus, this paper is a continuation of the previous papers (Śloderbach, [1], [2], [3], [8], [30]). The conditions of uniqueness and bifurcation criteria derived in the previous papers (Śloderbach, [1], [3]) concerned a comparison body derived for generalized coupled thermoplasticity depending on kinematically admissible strain velocity fields.

3. We can assume that in the areas of the plastically deformed body where the conditions of uniqueness or the bifurcation criteria are exceeded, submicro- or microconcentrations of strains can occur. Microcracks and microlocalizations of strains are possible; then they become macrolocalizations while further developing and nucleation occurs, leading to a crack in the material. The influence of such concentrators can be especially important under variable mechanical and thermomechanical loadings, or creep, so connected with fatigue strength or material cracking (Nguyen, [27]; Raniecki and Sawczuk, [36]; Śloderbach, [30]; Śloderbach and Pajak, [28]).

4. In a generalized case, constitutive equations of coupled thermoplasticity are of the character of nonassociated laws of plastic flow, even in the case of the assumption of Gyarmati postulate (Gyarmati [9]), see (Śloderbach, [1], [2], [8], [30]). These equations include effects of thermomechanical couplings, and include a phenomenon of elastic-plastic coupling. It means that they can be applied for a description of not only plastic metallic, brittle and semi-brittle materials, but porous materials, sintered powders, rocks, soils, concretes and other materials as well (Hueckel and Maier, [39], [40]; Maier, [41]).

5. In the paper, only general expressions were derived for constitutive functions of coupled generalized thermoplasticity. They occur in both necessary and sufficient global and local conditions of uniqueness of solution of the formulated incremental boundary-value problem. During further investigations, the constitutive functions of coupled generalized thermoplasticity should be specified within mechanics of continuous media according to experimental results.

\section{Nomenclature}

$c_{\varepsilon}$ and $c_{\sigma} \quad$ - specific heat capacity measured at constant elastic strain and stresses in $[1 /(\mathrm{kgK})]$

$D$ - expresses dissipation of mechanical energy per unit time and volume

$\operatorname{div} \boldsymbol{q}=\frac{\partial q_{i}}{\partial x i}, x_{i}-$ orthogonal coordinates which express the initial location of the particle

E - Young's modulus

$\boldsymbol{e}^{p}-$ deviatoric part of plastic deformation tensors $\boldsymbol{\varepsilon}^{p}, \boldsymbol{e}^{p} \equiv \operatorname{dev} \varepsilon^{p}$

$F$ - law function of plastic flow determined in the variables state space $\{T, \sigma, K\}$

$F_{l}$ - generalized law function of plastic flow determined in the thermodynamic force space $\{T, \sigma,-\Pi, K\}$

$h$ - hardening function

$K \quad$ - pair of internal parameters $K \Leftrightarrow\left\{\boldsymbol{\kappa}^{(\boldsymbol{M})} ; \boldsymbol{\kappa}^{(N)}\right\}_{M=1} \quad m, N=1 \quad n$

$\boldsymbol{M}$ and $\boldsymbol{L}$ - tensor of isothermal elastic moduli and tensor of elastic compliance, respectively

$$
\left.\left(\boldsymbol{M}=\boldsymbol{L}_{l}^{-1}\right)\right|_{\sigma=\sigma(T, \varepsilon, K)} \text {, and } 2\left(M_{i j m n} L_{m n r s}\right)_{\sigma=\sigma\left(Y_{K}^{T \varepsilon}\right)}=\delta_{i s} \delta_{j r}+\delta_{i r} \delta_{j s} \text { and } M_{i j m \boldsymbol{n}}=M_{\boldsymbol{m} \boldsymbol{n i j}}=M_{j i m \boldsymbol{n}}=M_{i j n \boldsymbol{m}}
$$


$\boldsymbol{N}$ - vector of pairs of tensors of the fourth and second order representing isothermal variation of the state of stress due to internal processes accompanying plastic deformation also in the state $Y_{K}^{T \varepsilon}$

$q$ - vector of the density of heat flow rate, $\left[\mathrm{J} /\left(\mathrm{m}^{2} \mathrm{~s}\right)\right]$

$q$ - denotes the heat flux exchange with the neighbourhood per unit time across an unit area in $\left[\mathrm{J} /\left(\mathrm{m}^{2} \mathrm{~s}\right)\right]$

$T$ - thermodynamic temperature in $[K]$

$T_{0}$ - reference temperature corresponding to the TRS - it may be, for example, the ambient temperature

$\nabla T$ - gradient $T(\operatorname{grad} T)$

(TRS) - abbreviation for "thermodynamic reference state", where $T=T_{0}, K=0$ and $\varepsilon^{e}=\mathbf{0}$

$X^{D} \quad$ set of dissipative (mechanical) thermodynamic forces, $X^{D}=\left\{\sigma,-\Pi, \frac{1}{T} \nabla T\right\}$

$\dot{\boldsymbol{x}}^{D} \quad$ - set of dissipative (mechanical) thermodynamic flows, $\dot{\boldsymbol{x}}^{D}=\left\{\dot{\boldsymbol{\varepsilon}}^{p}, \boldsymbol{q}, \dot{K}\right\}$

$Y-$ yield stress in uniaxial tension

$Y_{0} \quad$ - initial yield stress, for $\boldsymbol{\varepsilon}^{p}=\mathbf{0}$

$Y_{1}-$ yield stress in uniaxial tension as dependent on $(\pi, \kappa, T)$

$Y_{\Pi}^{T \varepsilon}=\left\{T, \varepsilon^{\mathrm{e}}, \Pi\right\}$

and $Y_{\Pi}^{T \sigma}=\{T, \sigma, \Pi\}$

- variables of thermodynamic state

$\boldsymbol{Z}$ - vector of tensor pairs composed of the fourth and second order representing the isothermal variation of elastic deformation due to the internal processes accompanying plastic deformation in state $Y_{K}^{T \sigma}$, then $\boldsymbol{Z} \Leftrightarrow\left\{Z_{m n k l} ; Z_{m n}\right\}$

$\alpha-$ symmetric tensor of thermal expansion coefficients, such that $\alpha \delta_{i j}=$ const

$\delta^{\mathrm{s}}$ - amount of entropy generated within a unit volume over a unit time and referred to a given material particle

$\varepsilon \quad-$ tensor of total deformations, $\varepsilon=\varepsilon^{e}+\varepsilon^{p}$

$\varepsilon^{e}$ and $\varepsilon^{p} \quad$ - tensor of small elastic and plastic deformations

$\kappa$ and $\kappa-$ symmetric second rank tensor and scalar internal parameter, respectively

$\Lambda$ - plasticity multiplier

$\mu$ and $\lambda-$ Lamé elastic constants

$v$ - Poisson ratio

$\Pi$ - pair of internal thermodynamic forces associated with a pair of internal parameters $K, \Pi \Leftrightarrow$ $\left\{\pi^{(M)}, \pi^{(N)}\right\}_{M=1 \quad m, N=1 n}$

$\rho_{0}$ and $\rho$ - body density in a thermodynamic reference state and in an actual one, respectively

$\sigma$ - Cauchy stress tensor

$\sigma_{(\mathrm{i})}-$ effective deviator of stress

$\sigma_{0}-$ yield stress value obtained in the uniaxial tension test for $\varepsilon^{p}=\mathbf{0}$

Tensors will be printed in a bold type-face. The summation convention is assumed along with the following detailed notation

$$
\begin{aligned}
& \mathbf{A B} \Leftrightarrow A_{i j} B_{j} \quad \text { or } \quad A_{i j k l} B_{k l} \quad(i, j, k, l, m, n, \ldots=1,2,3) \\
& \operatorname{tr} \mathbf{A} \Leftrightarrow A_{k k}, \quad \operatorname{tr}(\mathbf{A B}) \Leftrightarrow A_{i j} B_{j i} \\
& \mathbf{A}: \mathbf{B} \Leftrightarrow A_{i} B_{i} \quad \text { or } \quad A_{i j} B_{i j}
\end{aligned}
$$$$
\mathbf{A} \otimes \mathbf{B} \Leftrightarrow A_{i} B_{j} \quad \text { or } \quad A_{i j} B_{k l}
$$

1 - identity tensor, $\delta_{i j}$ - Kronecker delta, $\boldsymbol{0}$ - null tensor,

$$
\operatorname{sym} \mathbf{A} \Rightarrow \frac{1}{2}\left(A_{i j}+A_{j i}\right), \operatorname{dev} \mathbf{A}=\boldsymbol{A}-\frac{1}{3}(\operatorname{tr} \mathbf{A}) \mathbf{1}-\text { deviatoric part of tensor } \mathbf{A}
$$




$$
\begin{aligned}
& A_{i, j}=\frac{\partial A_{i}}{\partial x_{j}}, \text { where: } x_{j}-\text { coordinates of a material particle } \\
& \dot{A}=\frac{\partial A}{\partial t}, \text { where: }(t-\text { time }), \frac{\partial \boldsymbol{A}}{\partial \boldsymbol{B}} d \boldsymbol{B} \Rightarrow \frac{\partial A_{i j}}{\partial B_{k l}} d B_{k l}
\end{aligned}
$$

If $\boldsymbol{Z}$ denotes pairs of tensors of the fourth and the second order, then $\boldsymbol{Z} \Leftrightarrow\left\{Z_{m n k l} ; Z_{m n}\right\}$, and $\boldsymbol{M}$ is the tensor of the fourth order, then the operation $M Z$ is a pair of tensors of the fourth and the second order defined as follows

$$
\boldsymbol{M Z} \Leftrightarrow\left\{M_{i j m n} Z_{m n k l} ; M_{i j m n} Z_{m n}\right\}
$$

If $\Pi$ and $K$ denote pairs of tensors of the second and the zeroth order, then the operation $\Pi \cdot K$ produces a scalar, cf (Śloderbach, [1], [2], [9], [30]),

$$
\Pi \cdot K=\pi: \kappa+\pi \kappa=\pi_{i j} \kappa_{i j}+\pi \kappa .
$$

If the function $F$ is relative to $\Pi$ and $K$, then $F(\Pi)=F(\pi, \pi)$ and $F(K)=F(\kappa, \kappa)$, the derivatives of function $F$ with respect to a pair $\Pi$ and $K$ are defined as follows

$$
\frac{\partial F}{\partial \Pi}=\left\{\frac{\partial F}{\partial \pi}, \frac{\partial F}{\partial \pi}\right\} \quad \text { and } \quad \frac{\partial F}{\partial K}=\left\{\frac{\partial F}{\partial \mathbf{k}}, \frac{\partial F}{\partial \kappa}\right\} .
$$

The differentials of function $F$ with respect to the pairs $K$ and $\Pi$ of tensors of the second and the zeroth order produce the following form

$$
\frac{\partial F}{\partial \Pi} \cdot d \Pi=\left\{\frac{\partial F}{\partial \pi_{i j}} d \pi_{i j}, \frac{\partial F}{\partial \pi} d \pi\right\} \text { and } \frac{\partial F}{\partial K} \cdot d K=\left\{\frac{\partial F}{\partial \kappa_{i j}} d \kappa_{i j}, \frac{\partial F}{\partial \kappa} d \kappa\right\} .
$$

The differential of function $F$ with respect to the pair of tensors of the second and the zeroth order, produces a sum, cf (Śloderbach, [1], [2], [9], [30]), such that

$$
\frac{\partial \Pi}{\partial \mathrm{K}} d \mathrm{~K} \Leftrightarrow\left\{\frac{\partial \pi_{i j}}{\partial \kappa_{k l}} d \kappa_{k l}+\frac{\partial \pi_{i j}}{\partial \kappa} d \kappa ; \frac{\partial \pi}{\partial \kappa_{k l}} d \kappa_{k l}+\frac{\partial \pi}{\partial \kappa} d \kappa\right\} .
$$

If $\boldsymbol{Z}$ denotes pairs of tensors of the fourth and the second order and $K$ is a pair of tensors of the second and the zeroth order, then the operation $\boldsymbol{Z} * \boldsymbol{K}$ produces a pair of tensors of the second order such that

$$
\boldsymbol{Z} * \boldsymbol{K} \Leftrightarrow\left\{Z_{i j m n} \kappa_{m n} ; Z_{i j} \kappa\right\}, \text { and }\left\{\begin{array}{l}
-\boldsymbol{L} \boldsymbol{N}=-\boldsymbol{L}\left(Y_{K}^{T \sigma}\right) \boldsymbol{N}\left(Y_{K}^{T \varepsilon}\right)=\boldsymbol{Z} \\
-\boldsymbol{M Z}=-\boldsymbol{M}\left(Y_{K}^{T \varepsilon}\right) \boldsymbol{Z}\left(Y_{K}^{T \sigma}\right)=\boldsymbol{N}
\end{array}\right.
$$

If $\alpha$ is a second order tensor, then the operation $\alpha \circ(\boldsymbol{M Z})$ produces a pair of tensors of the second and the zeroth order

$$
\boldsymbol{\alpha} \circ(\boldsymbol{M Z}) \Leftrightarrow\left\{\boldsymbol{\alpha}_{i j} M_{i j m n} Z_{m n k l} ; \boldsymbol{\alpha}_{i j} M_{i j m n} Z_{m n}\right\}
$$

\section{References}

[1] Śloderbach Z. (1980): Bifurcations criteria for equilibrium states in generalized thermoplasticity [in Polish], Ph.D. thesis. - IFTR Reports, Institute of Fundamental Technological Research, Polish Academy of Sciences, nr 37/1980, Warsaw, pp.1-100.

[2] Śloderbach Z. (1983): Generalized coupled thermoplasticity. Part I. Fundamental equations and identities. Archives of Mechanics, No.35, vol.3, pp.337-349. 
[3] Śloderbach Z. (1983): Generalized coupled thermoplasticity. Part II. On the uniqueness and bifurcations criteria. Archives of Mechanics, No.35, vol.3, pp.351-367.

[4] Mróz Z. and Raniecki B. (1976): A derivation of the uniqueness conditions in coupled thermoplasticity. - Int. J. Eng. Sci., No.14, pp.395-401.

[5] Mróz Z. and Raniecki B. (1976): On the uniqueness problem in coupled thermoplasticity. - Int. J. Eng. Sci., No.14, pp.211-221.

[6] Raniecki B. (1977): Problems of Applied Thermoplasticity [in Polish], DSc Thesis. - IFTR Reports, Institute of Fundamental Technological Research, Polish Academy of Sciences, nr 29/1977, Warsaw, pp.1-120.

[7] Śloderbach Z. (2016): Closed set of the uniqueness conditions and bifurcations criteria in generalized coupled thermoplasticity for small deformations. - Continuum Mechanics and Thermodynamics, vol.28, pp.633-654.

[8] Śloderbach Z. (2016): Closed system of coupling effects in generalized thermo-elastoplasticity. - International Journal of Applied Mechanics and Engineering, vol.21, No.2, pp.461-483.

[9] Gyarmati J. (1970): Non-equilibrium thermodynamics. Field theory and variational principles. - Berlin, New York: Springer-Verlag.

[10] Armero F. and Simo J.C. (1993): A priori estimates and unconditionelly stable product formula alghorithms for nonlinear coupled thermoplasticity. - Int. J. Plasticity, vol.9, pp.749-782.

[11] Benall A. and Bigoni D. (2004): Effects of temperature and thermo-mechanical couplings on material instabilities and strain localization of inelastic materials. - J. Mech. Phys. Solids, vol.52, No.3, pp.725-753.

[12] Bertram A. (2003): A finite thermoplasticity based on isomorphisms. - Int. J. Plasticity, vol.19, pp.2027-2050.

[13] Candija M. and Brnic J. (2004): Associative coupled thermoplasticity at finite strain with temperature-dependent material parameters. - Int. J. Plasticity, vol.20, No.10, pp.1851-1874.

[14] Casey J. (1998): On elastic-thermo-plastic materials at finite deformations. - Int. J. Plasticity, vol.14, No.1-3, pp.173-191.

[15] Epstein M. and Maugin G.A. (2000): Thermomechanics of volumetyric growth in uniform bodies. - Int. J. Plasticity, vol.16, No.7-8, pp.951-978.

[16] Hakansson P., Wallin M. and Ristinmaa M. (2002): Comparison of isotropic hardening and kinematic hardening in thermoplasticity. - Int. J. Plasticity, vol.18, pp.379-397.

[17] Huttel C. and Matzenmiller A. (1999): Extension of generalized plasticity to finite deformations and structures. Int. J. Plasticity, vol.36, pp.5255-5276.

[18] Itskov M. (2004): On the application of the additive decomposition of generalized strain measures in large strain plasticity. - Mechanics Res. Commun., vol.31, No.5, pp.507-517.

[19] Lehmann Th. (1991): Thermodynamical foundations of large inelastic deformations of solid bodies including damage. - Int. J. Plasticity, vol.7, pp.79-98.

[20] Mahnken R. and Stein E. (1997): Parametr identification for finite deformation elasto-plasticity in principal directions. - Computer Method Applied Mech. Engineering, vol.147, No.1-2, July 30, pp.17-39.

[21] Marakin A.A. and Sokolova M.Y. (2002): Thermomechanical models of irreversible finite deformation of anisotropic solids, strength of materials. - Vol.34, No.6, pp.529-535.

[22] Maugin G.A. and Epstein M. (1998): Geometrical material structure of elastoplasticity. - Int. J. Plasticity, vol.14, pp.109-115.

[23] Meyers A., Bruhns O.T. and Xiao H. (2000): Large strain response of kinematic hardening elastoplasticity with the logarythmic rate: Swift effect in torsion. - Meccanica, vol.35, No.3, pp.229-247.

[24] Miehe C. (1995): A theory of large strain isotropic thermoplasticity based on metric transformation tensors. Archive of Applied Mechanics, vol.66, No.1/2, pp.45-64. 
[25] Srinivasa A.R. (2001): Large deformation plasticity and the poynting effect.- Int. J. Plasticity, vol.17, No.9, pp.1189-1214.

[26] Xiao H., Bruhns O.T. and Meyers A. (2007): Thermodynamic laws and consistent Eulerian formulation of finite elastoplasticity with thermal effects. - J. Mech. Phys. Solids, vol.55, No.2, pp.338-365.

[27] Nguyen H.V. (1999): Thermomechanical Couplings in Elasto-Plastic Metals in the Case of Finie Deformations, DSc Thesis [in Polish]. - IFTR-Reports, No 10/1999, pp.1-116, Institute of Fundamental Technological Research, Polish Academy of Sciences, Warsaw.

[28] Śloderbach Z. and Pajak J. (2010): generalized coupled thermoplasticity taking into account large strains: Part I. Conditions of uniqueness of the solution of boundary-value problem and bifurcation criteria. - Mathematics and Mechanics of Solids, vol.15, No.3, pp.308-327.

[29] Śloderbach Z., Pajak J. (2010): Generalized coupled thermoplasticity taking into account large strains: Part II. A model of non-compressible elastic-plastic solid with non-associated plastic flow law. - Mathematics and Mechanics of Solids, vol.15, No.3, pp.28-341.

[30] Śloderbach Z. (2014): Thermomechanical problems of deformable bodies for small deformations. - Opole University of Technology, ISBN 978-8364056-54-3, pp.1-272.

[31] Maugin G.A. (1990): Internal variables and dissipative structure. - J. of Non-Equilibrium Thermodynamics, vol.15, pp.173-192.

[32] Simo J. and Miehe C. (1992): Associative coupled thermoplasticity at finite strains: formulation, numerical analysis and implementation. - Computer Method Applied Mech. Engineering, vol.98, pp.41-104.

[33] Raniecki B. (1979): Uniqueness criteria in solids with non-associated plastic flow laws at finite deformation. Bulletin De l'Acadademie Polonaise des Sciences, Serie des Sciences Techniques, vol.27, pp.391-399.

[34] Raniecki B. and Bruhns O.T. (1981): Bounds to bifurcation stress in solids with non-associated plastic flow at finite strain. - J. Mech. Phys. Solids, vol.29, No.2, pp.153-172.

[35] Raniecki B. and Sawczuk A. (1975) Thermal effects in plasticity. Part I: Coupled theory. - Zeitschrift für Angewandte Mathematik und Mechanik - ZAMM, vol.55, No.6, pp.232-241.

[36] Raniecki B. and Sawczuk A. (1975): Thermal effects in plasticity. Part II: Uniqueness and applications. Zeitschrift für Angewandte Mathematik und Mech.-ZAMM, vol.55, No.7/8, pp.363-373.

[37] Mróz Z. (1963): Non-associated flow laws in plasticity. - J. de Mecanique, vol.2, No.1, pp.21-42.

[38] Mróz Z. (1966): On forms of constutive laws for elastic-plastic solids. - Arch. Applied Mechanics, vol.18, No.1, Warszawa, pp.3-35.

[39] Hueckel T. and Maier G. (1977): Incremental boundary value problems in the presence of coupling of elestic and plastic deformations. A rock mechanics oriented theory. - International Journal of Solids and Structures, vol.13, pp.1-15.

[40] Hueckel T. and Maier G. (1977): Non-associated and coupled flow rules of elastoplasticity for geotechnical media. - Proc. 9-th International Conference of Soil Mechanics Foundation of Engineering (JCSFE), Tokyo, Speciality Session 7, Constutive relations for soils, pp.129-142.

[41] Maier G. (1970): A minimum principle for incremental elastoplasticity, with non-associated flow laws. - Journal Mechanics of Physics Solids, vol.18, pp.319-330.

Received: February 3, 2016

Revised: June 30, 2016 